\title{
Conduit convection driving persistent degassing at basaltic volcanoes
}

\author{
F.M. Beckett ${ }^{\text {a,* }}$, M. Burton ${ }^{\text {b }}$, H.M. Mader ${ }^{\text {a }}$, J.C. Phillips ${ }^{\text {a }}$, M. Polacci ${ }^{\text {b }}$, A.C. Rust ${ }^{\text {a }}$, F. Witham ${ }^{\text {a, } 1}$ \\ a Department of Earth Sciences, University of Bristol, Wills Memorial Building, Queens Road, Bristol BS8 1RJ, UK \\ ${ }^{\mathrm{b}}$ Istituto Nazionale di Geofisica e Vulcanologia, Sezione di Pisa, via della Faggiola 32, 56126 Pisa, Italy
}

\section{A R T I C L E I N F O}

\section{Article history:}

Received 26 January 2014

Accepted 10 June 2014

Available online 2 July 2014

\section{Keywords:}

Volcanology

Stromboli

Persistent degassing

Magma convection

\begin{abstract}
A B S T R A C T
The persistent release of gas at basaltic volcanoes where there is a low magma eruption rate can be driven by an exchange flow of magma in the conduit, in which gas-rich magma ascends, degasses and crystallises and then sinks back down the conduit. The driving force of the flow is provided by the density difference between the buoyant bubble-rich magma at depth and the dense degassed crystallised magma at shallow levels. In this study we attempt to constrain the physical and chemical processes driving an exchange flow of magma at Stromboli, Aeolian Archipelago, Italy. The model uses a simple, cylindrical geometry. We define degassing and crystallisation paths of the ascending and descending magmas, constrained by gas flux and melt inclusion data given in the literature, to produce a three-phase model of ascending and descending magmas driving persistent gas fluxes. We calculate the viscosity of the three-phase magma using end-member rheological models for bubble and crystal suspensions. Combining our modelled magma properties with analogue exchange flow experiments we can relate the regime of magma flow driving persistent degassing to pressure. At pressures $\$ 90 \mathrm{MPa}$ $(\leqslant 3 \mathrm{~km}$ ) the viscosity ratio is $\lesssim 100$ and the regime is predicted to be side by side flow with both ascending and descending magmas adjacent to a portion of the conduit wall. At pressures $\gtrsim 90 \mathrm{MPa}$ ( $\gtrsim 3 \mathrm{~km}$ ) the viscosity ratio between the ascending and descending magma is $\gtrsim 100$ and the flow is predicted to be core annular flow, with the ascending vesiculating magma in the inner core and the more crystalline degassed magma flowing down along the conduit wall. By analogy, we hypothesise that degassed magma would flow down along the walls in dike-like plumbing geometries that have been proposed for the deeper Strombolian system. Analogue experiments suggest that exchange flows do not overturn under conditions of maximum volume flux; we use an empirical relationship to characterise the volume flux of the exchange flow and show that the radius of a cylindrical conduit required to account for the observed persistent gas flux at Stromboli at $0.1 \mathrm{MPa}$ is $\sim 1 \mathrm{~m}$.
\end{abstract}

(c) 2014 Elsevier B.V. All rights reserved.

\section{Introduction}

Persistent gas fluxes at many active basaltic volcanoes such as Etna (Sicily, Italy), Stromboli (Aeolian Archipelago, Italy), Izu Oshima (Japan) and Masaya (Nicaragua) cannot be reconciled with the exclusive degassing of magma erupted during effusive and explosive episodes. At Etna, Allard (1997) estimates that $~ 80-90 \%$ of gas emissions are generated by magma that is never erupted. At Stromboli $\sim 50,000 \mathrm{t} \mathrm{d}^{-1}$ of magma must be degassed to account for the persistent gas flux of $\sim 200 \mathrm{t} \mathrm{d}^{-1}$ sulphur dioxide ( $\mathrm{SO}_{2}$ ) (Allard et al., 2008). Normal Strombolian explosive activity and effusive eruptions emit on average only $\sim 530 \mathrm{t} \mathrm{d}^{-1}$ of solid matter (Allard et al., 1994) and thus the extruded magma flux represents $\sim 1 \%$ of the magma processed during persistent degassing. To account for the continuous supply of gas and heat recorded, where there is no significant extrusion of magma, an exchange flow of magma within the conduit is inferred, driven by the

\footnotetext{
* Corresponding author at: Met Office, Fitzroy Road, Exeter EX1 3PB, UK

E-mail address: frances.beckett@metoffice.gov.uk (F.M. Beckett).

${ }^{1}$ Now at Combustion and Turbines, Rolls-Royce, Bristol BS34 7QE, UK.
}

density difference between dense degassed magma at shallow levels and buoyant gas-rich magma deeper within the system (Kazahaya et al., 1994; Harris and Stevenson, 1997; Stevenson and Blake, 1998). To interpret gas emissions and understand changes in activity at persistently active basaltic volcanoes therefore require some knowledge of the flow regime of the exchange flow, and how its volume flux relates to the degassing regimes observed and the physical properties of the magma: its viscosity and density and thus crystallinity, vesicularity and the composition of its melt.

Experimental studies of exchange flow in vertical cylindrical pipes, where the volume flux of the ascending fluid is matched by an equal flux of descending fluid, have shown that the flow regime of an exchange flow is a function of the viscosity ratio $\beta=\mu_{2} / \mu_{1}$, the viscosity of the more viscous fluid divided by the viscosity of the less viscous fluid (Stevenson and Blake, 1998; Huppert and Hallworth, 2007; Beckett et al., 2011). In this study we explore how to apply the experimental observations to a persistently degassing basaltic volcano. We use Stromboli as a case study as there are extensive data sets on the chemical composition of its magma and well constrained measurements of the flux and composition of the gas released. However, 
a) $\mathrm{CAF}$

b) SBS

c) TSBS
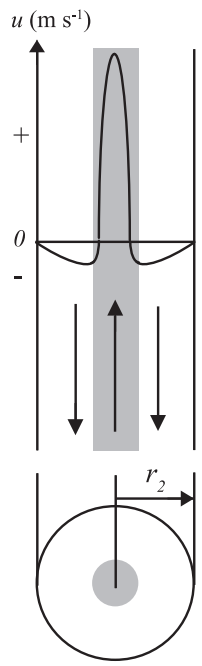

Plan View
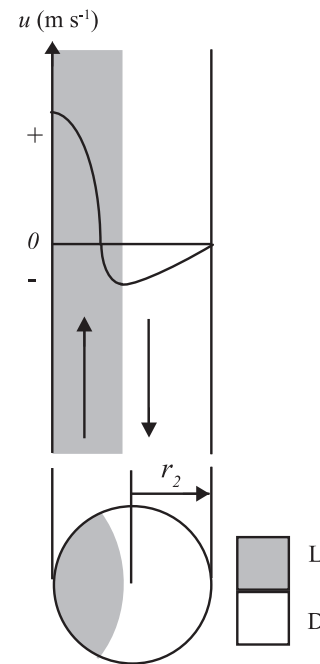

Less Dense, Less Viscous Fluid

Dense, Viscous Fluid

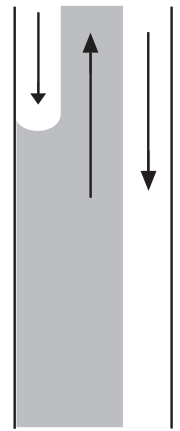

Fig. 1. Cartoon to show the flow types of buoyancy driven, laminar, exchange flow in a vertical pipe, a. Core annular flow (CAF), b. Side by side (SBS) flow and c. Transitional side by side (TSBS) flow. Arrows indicate the dominant flow direction, however at the fluid interface the less viscous fluid flows downwards, in the same direction as the more viscous fluid, as indicated by the velocity profiles for CAF and SBS flow.

application of the experimental observations requires that we know the density and viscosity of the magma as a function of pressure during both ascent and decent. Using melt inclusion (MI) data given by Métrich et al. (2010) and measured gas flux data (Burton et al., 2009), we use VolatileCalc (Newman and Lowenstern, 2002) and MELTS (Ghiorso and Sack, 1995; Asimow and Ghiorso, 1998) to constrain the crystallisation and degassing path of ascending and descending magma as a function of pressure. We then apply new methods to characterise the multiphase rheology of the ascending and descending magma, accounting for its crystallinity, vesicularity and melt composition. To calculate the rheology of the three-phase system we explore two approaches: in the first the viscosity of the bubble suspension is calculated taking the crystals plus melt as the suspending fluid; in the second the viscosity of a crystal suspension is calculated taking the bubbles plus melt as the suspending fluid. It is shown that there is no significant

difference in the calculated viscosities from the two methods. Using the calculated viscosity and density of the magma as a function of pressure, we determine the Reynolds number of an exchange flow at Stromboli and show that the flow is laminar, and thus we can apply experimental observations of low Reynolds number exchange flows. We calculate the viscosity ratio between the ascending and descending magma as a function of pressure and define the flow regime of an exchange flow at Stromboli. Although the degassing behaviour at Stromboli and the properties of its magma are well constrained, the geometry of its feeder system remains unclear and likely includes dyke-like structures (Chouet et al., 2003). All experimental studies of exchange flow have been conducted in cylindrical pipes and in order to apply the experimental results we assume a single cylindrical conduit, the most thermally efficient and mechanically robust geometry for a steady-state system. This conduit geometry is an over-simplification and we discuss the limitations of this approach and the implications for defining the flow regime of an exchange flow of magma at persistently degassing basaltic volcanoes.

\section{Background}

There have been a number of theoretical, numerical and experimental studies that have attempted to better understand how a density driven exchange flow can drive persistent gas fluxes at basaltic volcanoes (Kazahaya et al., 1994; Stevenson and Blake, 1998; Huppert and Hallworth, 2007; M.R. Burton et al., 2007; Beckett et al., 2011; Kerswell, 2011; Palma et al., 2011; Molina et al., 2012). Stevenson and Blake (1998) presented analogue experiments of simple transient exchange flows in which the flow regime in a vertical cylindrical pipe was found to be core annular flow (CAF), with the less viscous fluid ascending up the centre of the pipe and the more viscous fluid descending down the walls in an outer annulus. Imposing a CAF geometry they present a theoretical model to constrain the conduit radius required to drive gas fluxes by assuming that the overturn rate of magma is proportional to the gas flux. Dimensional analysis shows that in a cylindrical conduit magma exchange flux is proportional to the conduit radius to the fourth power, and thus the radius of the conduit is highly insensitive to the volume flux of the exchange flow. The properties of the exchanging fluids are estimated assuming that one represents magma in a chamber at a depth of $2 \mathrm{~km}$ and the other a shallow degassed magma. They take typical properties for basaltic magma and assume that properties are independent of pressure for each fluid and so do not account for degassing and crystallisation paths, such that ascending magma has a water content of 0.5 wt.\% and a crystallinity of 30 vol.\%. They a)

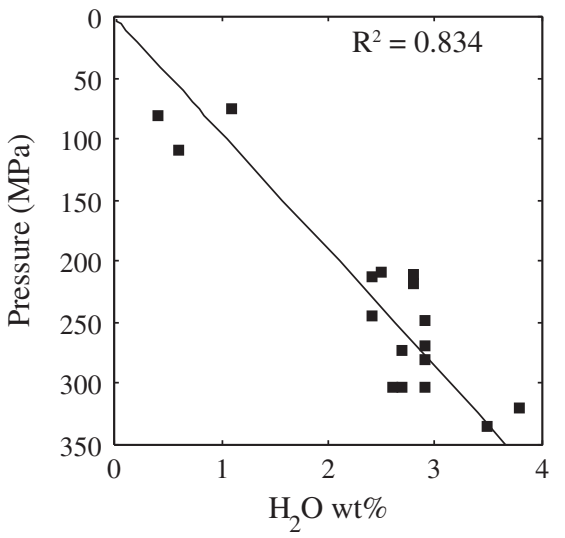

b)

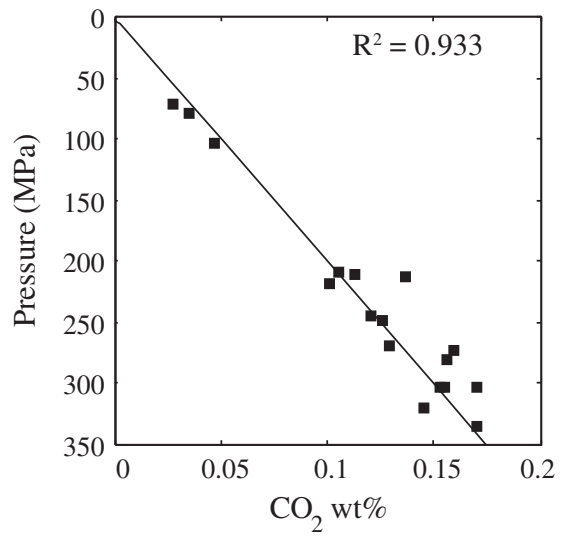

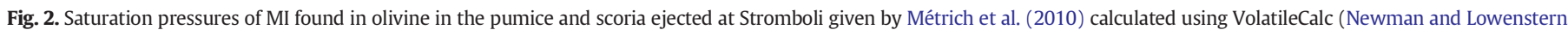
2002) assuming closed-system degassing. Simple linear fits allow us to define the degassing path, such that a. $\mathrm{X}_{\mathrm{H}_{2} \mathrm{O}}=0.0105 \cdot P$ and $\mathrm{b} . \mathrm{X}_{\mathrm{CO}_{2}}=0.0005 \cdot P$. 
conclude that to account for a water mass flux of $\sim 70 \mathrm{~kg} \mathrm{~s}^{-1}$, calculated from the $\mathrm{SO}_{2}$ flux and weighted ratios of $\mathrm{H}_{2} \mathrm{O}, \mathrm{CO}_{2}, \mathrm{HCl}$ and $\mathrm{HF}$ to $\mathrm{SO}_{2}$ from analysis of both fumarolic and quiescent plume emissions given by Allard et al. (1994), the radius of the conduit at Stromboli has to be 4-10 m.

M.R. Burton et al. (2007) provide a more quantitative determination of the density difference driving overturn at Stromboli. They use constraints from the literature on the petrology and texture of erupted products as well as $\mathrm{SO}_{2}$ flux data to define the degassing path of ascending magma as a function of pressure, but do not define its crystallisation path. They show that simple closed-system degassing of primitive basalt at Stromboli results in gas volume fractions of over 99.9\%, which is incompatible with the vesicularity $\left(\phi_{b}\right)$ of scoria ejected during normal Strombolian eruptions, $\phi_{b}=0.3-0.8$ (Polacci et al., 2009). They assume that at a vesicularity of 50 vol.\%, which they find to occur at 50$100 \mathrm{MPa}$, there is a transition from closed- to open-system degassing where the magma becomes permeable to gas flow with the gas then rising separately from the magma. They present a conduit flow model to calculate the velocities of the ascending and descending magmas using density differences from their calculated density profiles. However, they use end-member values for the viscosities of the ascending and descending magmas $\left(\mu_{1,2}\right)$ given by Métrich et al. (2001): $\mu_{1} \sim 20-30$ Pa s for an original un-crystallised magma and $\mu_{2} \sim 14,000$ Pa $\mathrm{s}$ for a degassed, 50 vol.\% crystallised magma. They assume a CAF geometry and that the ascending magma occupies a radius such as to maximise the exchange flux, an assumption shown to be invalid by analogue exchange flow experiments conducted by Beckett et al. (2011). They conclude that the conduit radius required to account for the mass flow rate of ascending magma (constrained by gas flux data) is $1.28 \mathrm{~m}$ at $50 \mathrm{MPa}$, which increases to $1.45 \mathrm{~m}$ at $200 \mathrm{MPa}$ in order to satisfy mass conservation.

Recent experiments by Huppert and Hallworth (2007), Beckett et al. (2011) and (Palma et al., 2011) have included fluid reservoirs at the ends of the pipes which allow longer exchange flow durations, representative of steady state conditions. Huppert and Hallworth (2007) performed experiments with $10^{-4}<R e_{2}<10^{1}$, where $R e_{2}$ is the Reynolds number of the more viscous fluid calculated given the volume flux of the flow, and $\beta$ ranging from $10^{0}$ to $10^{5}$. They state that the predominant behaviour is CAF, with the less viscous fluid occupying the core, but also note that 'the fluid may be highly irregular and break into discrete blobs or partially adhere to the side wall of the conduit'. Palma et al. (2011) conducted experiments in vertical and inclined cylindrical pipes, at viscosity ratios $(\beta)$ from 3.38 to $1.83 \times 10^{3}$, and Reynolds numbers $10^{-4}-10^{1}$. In pipes inclined at $6^{\circ}$ and $20^{\circ}$ to vertical, they observed a stratified flow pattern, where the dense fluid is in contact with the lower side of the pipe and the lighter fluid is in contact with the upper side of the pipe, noting that exchange flows at the lower inclination appeared unstable. In vertical pipes, they observed CAF and 'pseudo-stratified' flow (although noting that these flow patterns were unstable), turbulent flow and 'blobs of lighter fluid rising through the continuous dense fluid'. They defined 'pseudo-stratified' flow as a similar flow pattern to stratified flow in inclined pipes. Huppert and Hallworth (2007) and Palma et al. (2011) analysed the flux of their exchange flows in terms of a 'flux coefficient' (the ratio of Reynolds to Grashof numbers), and Palma et al. (2011) used experimental results to identify flux coefficients for vertical and inclined cylindrical pipe flows. They also proposed that the same analysis could be applied to flows in multiple cylindrical or slot-shaped conduits through the use of an 'equivalent radius', but did not present experimental results to identify flux coefficient values corresponding to these configurations.

Palma et al. (2011) applied their experimental results to degassing at Villarica volcano, using the magma properties and the melt viscosity model of Hui and Zhang (2007), and the Einstein-Roscoe equation to account for the effect of crystals on the magma viscosity. The volumetric exchange flow rate was obtained from the magma properties, measurements of sulphur outgassing and the initial sulphur content of melt inclusions, and the experimentally-determined flux coefficients. A range

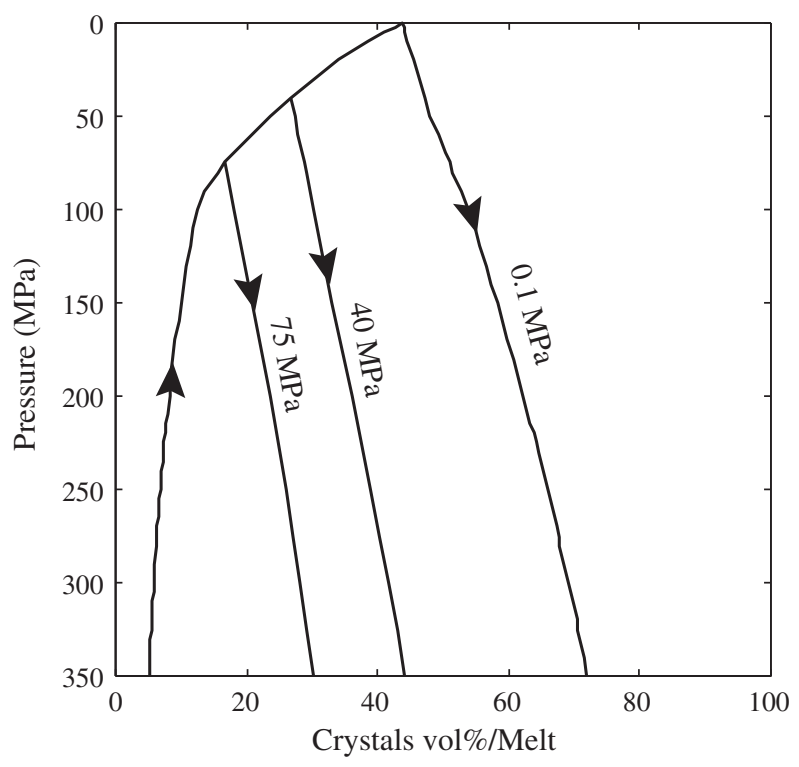

Fig. 3. The crystallisation paths of ascending and descending magma at Stromboli, modelled using MELTS (Ghiorso and Sack, 1995; Asimow and Ghiorso, 1998) with the bulk composition of pumice ST82 (Métrich et al., 2010). The wt.\% of $\mathrm{H}_{2} \mathrm{O}$ is inputted into the model at each pressure using the $\mathrm{X}_{\mathrm{H}_{2} \mathrm{O}}-P$ fit given in Fig. 2. Crystallisation paths of descending magma are shown assuming that magma ascends to $0.1 \mathrm{MPa}, 40 \mathrm{MPa}$ and $75 \mathrm{MPa}$ in the system.

of different initial volatile contents and magma properties was used in the calculations, but the properties of the rising and degassed magma were taken to be constant during transport. Palma et al. (2011) identified volatile content and magma property ranges that produced outgassing fluxes comparable to observations at Villarica.

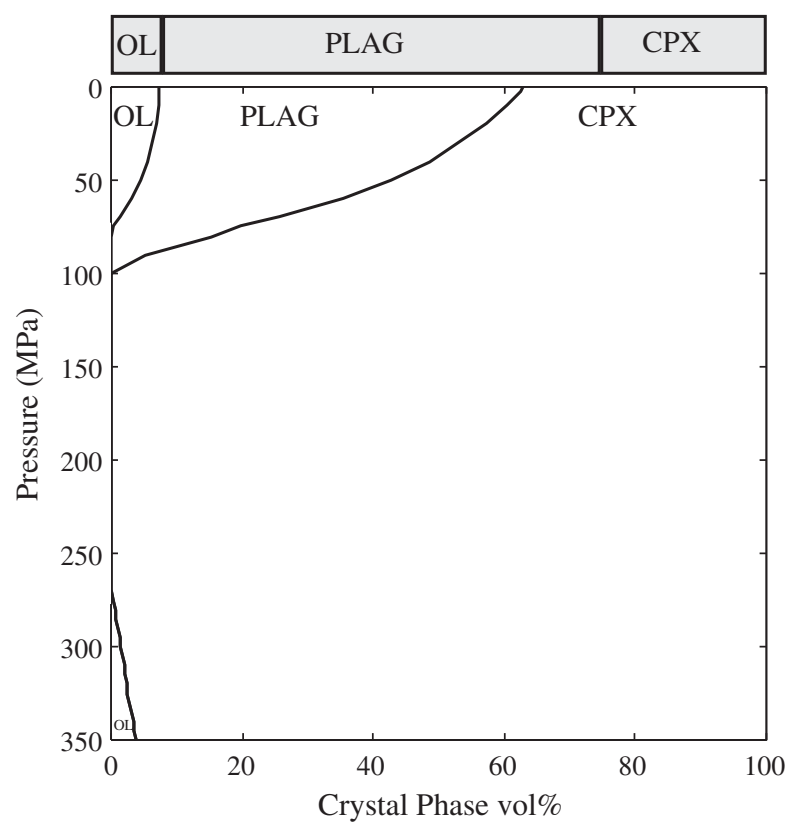

Fig. 4. Volume percent of plagioclase (PLAG), clinopyroxene (CPX) and olivine (OL) in the ascending magma at Stromboli determined from MELTS (Ghiorso and Sack, 1995; Asimow and Ghiorso, 1998) using the bulk composition of pumice ST82 (Métrich et al., 2010), calculated at $\mathrm{T}=1130{ }^{\circ} \mathrm{C}$ and $\mathrm{fO}_{2}$ fixed at $\triangle \mathrm{NNO}+1$. The average crystal content of scoria ejected at Stromboli, after Métrich et al. (2001), Francalanci et al. (2004), Landi et al. (2004), and Métrich et al. (2010), are shown for comparison, indicated by the grey box. 
Table 1

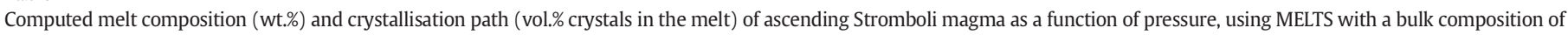
pumice ST82 as given by Métrich et al. (2010) for T $=1130{ }^{\circ} \mathrm{C}$.

\begin{tabular}{|c|c|c|c|c|c|c|c|c|c|c|c|c|c|}
\hline \multicolumn{14}{|c|}{ Bulk composition } \\
\hline Input $\mathrm{H}_{2} \mathrm{O}$ & & 3.68 & 3.62 & 3.57 & 3.52 & 3.47 & 3.41 & 3.36 & 3.31 & 3.26 & 3.20 & 3.15 & 3.10 \\
\hline$P(\mathrm{MPa})$ & & 350 & 345 & 340 & 335 & 330 & 325 & 320 & 315 & 310 & 305 & 300 & 295 \\
\hline $\mathrm{SiO}_{2}$ & 50.9 & 49.49 & 49.52 & 49.54 & 49.57 & 49.60 & 49.63 & 49.65 & 49.68 & 49.71 & 49.73 & 49.76 & 49.79 \\
\hline $\mathrm{TiO}_{2}$ & 0.9 & 0.89 & 0.89 & 0.89 & 0.89 & 0.89 & 0.89 & 0.89 & 0.89 & 0.89 & 0.89 & 0.89 & 0.89 \\
\hline $\mathrm{Al}_{2} \mathrm{O}_{3}$ & 16.6 & 16.88 & 16.90 & 16.91 & 16.93 & 16.95 & 16.97 & 16.99 & 17.00 & 17.02 & 17.04 & 17.06 & 17.08 \\
\hline $\mathrm{Fe}_{2} \mathrm{O}_{3}$ & & 2.34 & 2.34 & 2.35 & 2.35 & 2.36 & 2.36 & 2.37 & 2.37 & 2.38 & 2.38 & 2.39 & 2.39 \\
\hline $\mathrm{FeO}$ & 8.22 & 5.92 & 5.93 & 5.92 & 5.92 & 5.92 & 5.92 & 5.92 & 5.92 & 5.92 & 5.92 & 5.92 & 5.92 \\
\hline $\mathrm{MnO}$ & 0.16 & 0.17 & 0.17 & 0.17 & 0.17 & 0.17 & 0.17 & 0.17 & 0.17 & 0.17 & 0.17 & 0.17 & 0.17 \\
\hline $\mathrm{MgO}$ & 6.66 & 5.80 & 5.80 & 5.80 & 5.80 & 5.80 & 5.80 & 5.80 & 5.80 & 5.80 & 5.80 & 5.80 & 5.80 \\
\hline $\mathrm{CaO}$ & 10.9 & 9.94 & 9.93 & 9.93 & 9.92 & 9.91 & 9.90 & 9.90 & 9.89 & 9.88 & 9.87 & 9.86 & 9.85 \\
\hline $\mathrm{Na}_{2} \mathrm{O}$ & 2.39 & 2.46 & 2.47 & 2.47 & 2.48 & 2.48 & 2.48 & 2.49 & 2.49 & 2.49 & 2.50 & 2.50 & 2.50 \\
\hline $\mathrm{K}_{2} \mathrm{O}$ & 1.82 & 1.88 & 1.89 & 1.89 & 1.89 & 1.90 & 1.90 & 1.90 & 1.90 & 1.91 & 1.91 & 1.91 & 1.92 \\
\hline $\mathrm{P}_{2} \mathrm{O}_{5}$ & 0.4 & 0.41 & 0.42 & 0.42 & 0.42 & 0.42 & 0.42 & 0.42 & 0.42 & 0.42 & 0.42 & 0.42 & 0.42 \\
\hline $\mathrm{H}_{2} \mathrm{O}$ & & 3.81 & 3.76 & 3.71 & 3.66 & 3.61 & 3.56 & 3.51 & 3.46 & 3.41 & 3.36 & 3.31 & 3.26 \\
\hline $\mathrm{CaO} / \mathrm{Al}_{2} \mathrm{O}_{3}$ & & 0.59 & 0.59 & 0.59 & 0.59 & 0.59 & 0.58 & 0.58 & 0.58 & 0.58 & 0.58 & 0.58 & 0.58 \\
\hline Olivine vol.\% & & 0.19 & 0.17 & 0.16 & 0.15 & 0.14 & 0.13 & 0.12 & 0.11 & 0.10 & 0.08 & 0.07 & 0.07 \\
\hline Cpx vol.\% & & 4.75 & 4.87 & 4.92 & 5.00 & 5.08 & 5.18 & 5.24 & 5.33 & 5.42 & 5.51 & 5.60 & 5.70 \\
\hline Plag vol.\% & & 0.00 & 0.00 & 0.00 & 0.00 & 0.00 & 0.00 & 0.00 & 0.00 & 0.00 & 0.00 & 0.00 & 0.00 \\
\hline Vol.\% crystals & & 4.94 & 5.04 & 5.09 & 5.15 & 5.22 & 5.31 & 5.36 & 5.43 & 5.51 & 5.59 & 5.68 & 5.76 \\
\hline Fo\% & & 85.90 & 85.90 & 85.90 & 85.90 & 85.90 & 85.90 & 85.90 & 85.90 & 85.90 & 85.90 & 85.90 & 85.90 \\
\hline
\end{tabular}

An\%

Bulk composition

\begin{tabular}{|c|c|c|c|c|c|c|c|c|c|c|c|c|c|}
\hline Input $\mathrm{H}_{2} \mathrm{O}$ & & 3.05 & 2.99 & 2.94 & 2.89 & 2.84 & 2.78 & 2.73 & 2.68 & 2.63 & 2.57 & 2.52 & 2.47 \\
\hline$P(\mathrm{MPa})$ & & 290 & 285 & 280 & 275 & 270 & 265 & 260 & 255 & 250 & 245 & 240 & 235 \\
\hline $\mathrm{SiO}_{2}$ & 50.9 & 49.81 & 49.84 & 49.87 & 49.90 & 49.93 & 49.96 & 49.99 & 50.02 & 50.05 & 50.08 & 50.11 & 50.15 \\
\hline $\mathrm{TiO}_{2}$ & 0.9 & 0.89 & 0.90 & 0.90 & 0.90 & 0.90 & 0.90 & 0.90 & 0.90 & 0.90 & 0.90 & 0.90 & 0.90 \\
\hline $\mathrm{Al}_{2} \mathrm{O}_{3}$ & 16.6 & 17.10 & 17.12 & 17.15 & 17.17 & 17.19 & 17.21 & 17.24 & 17.26 & 17.28 & 17.31 & 17.34 & 17.37 \\
\hline $\mathrm{Fe}_{2} \mathrm{O}_{3}$ & & 2.40 & 2.40 & 2.41 & 2.41 & 2.42 & 2.42 & 2.43 & 2.43 & 2.44 & 2.44 & 2.45 & 2.45 \\
\hline $\mathrm{FeO}$ & 8.22 & 5.92 & 5.92 & 5.92 & 5.92 & 5.92 & 5.92 & 5.92 & 5.92 & 5.92 & 5.92 & 5.92 & 5.91 \\
\hline $\mathrm{MnO}$ & 0.16 & 0.17 & 0.17 & 0.17 & 0.17 & 0.17 & 0.17 & 0.17 & 0.17 & 0.17 & 0.17 & 0.17 & 0.17 \\
\hline $\mathrm{MgO}$ & 6.66 & 5.80 & 5.79 & 5.79 & 5.79 & 5.79 & 5.78 & 5.77 & 5.76 & 5.76 & 5.74 & 5.73 & 5.72 \\
\hline $\mathrm{CaO}$ & 10.9 & 9.84 & 9.83 & 9.82 & 9.81 & 9.79 & 9.78 & 9.77 & 9.76 & 9.76 & 9.74 & 9.73 & 9.71 \\
\hline $\mathrm{Na}_{2} \mathrm{O}$ & 2.39 & 2.51 & 2.51 & 2.52 & 2.52 & 2.52 & 2.53 & 2.53 & 2.54 & 2.54 & 2.55 & 2.55 & 2.56 \\
\hline $\mathrm{K}_{2} \mathrm{O}$ & 1.82 & 1.92 & 1.92 & 1.93 & 1.93 & 1.93 & 1.94 & 1.94 & 1.94 & 1.95 & 1.95 & 1.96 & 1.96 \\
\hline $\mathrm{P}_{2} \mathrm{O}_{5}$ & 0.4 & 0.42 & 0.42 & 0.42 & 0.42 & 0.42 & 0.43 & 0.43 & 0.43 & 0.43 & 0.43 & 0.43 & 0.43 \\
\hline $\mathrm{H}_{2} \mathrm{O}$ & & 3.21 & 3.16 & 3.11 & 3.06 & 3.01 & 2.96 & 2.91 & 2.86 & 2.81 & 2.76 & 2.71 & 2.66 \\
\hline $\mathrm{CaO} / \mathrm{Al}_{2} \mathrm{O}_{3}$ & & 0.58 & 0.57 & 0.57 & 0.57 & 0.57 & 0.57 & 0.57 & 0.57 & 0.57 & 0.56 & 0.56 & 0.56 \\
\hline Olivine vol.\% & & 0.05 & 0.04 & 0.02 & 0.02 & 0.00 & 0.00 & 0.00 & 0.00 & 0.00 & 0.00 & 0.00 & 0.00 \\
\hline Cpx vol.\% & & 5.79 & 5.90 & 6.01 & 6.10 & 6.23 & 6.34 & 6.45 & 6.56 & 6.64 & 6.79 & 6.91 & 7.04 \\
\hline Plag vol.\% & & 0.00 & 0.00 & 0.00 & 0.00 & 0.00 & 0.00 & 0.00 & 0.00 & 0.00 & 0.00 & 0.00 & 0.00 \\
\hline Vol.\% crystals & & 5.84 & 5.94 & 6.03 & 6.12 & 6.23 & 6.34 & 6.45 & 6.56 & 6.64 & 6.79 & 6.91 & 7.04 \\
\hline Fo\% & & 85.90 & 85.90 & 85.90 & 85.00 & 85.00 & & & & & & & \\
\hline
\end{tabular}

An\%

Bulk composition

\begin{tabular}{|c|c|c|c|c|c|c|c|c|c|c|c|c|c|}
\hline Input $\mathrm{H}_{2} \mathrm{O}$ & & 2.42 & 2.36 & 2.31 & 2.26 & 2.21 & 2.15 & 2.10 & 2.00 & 1.89 & 1.79 & 1.68 & 1.58 \\
\hline$P(\mathrm{MPa})$ & & 230 & 225 & 220 & 215 & 210 & 205 & 200 & 190 & 180 & 170 & 160 & 150 \\
\hline $\mathrm{SiO}_{2}$ & 50.9 & 50.18 & 50.21 & 50.24 & 50.28 & 50.31 & 50.35 & 50.38 & 50.45 & 50.52 & 50.60 & 50.68 & 50.75 \\
\hline $\mathrm{TiO}_{2}$ & 0.9 & 0.90 & 0.91 & 0.91 & 0.91 & 0.91 & 0.91 & 0.91 & 0.91 & 0.92 & 0.92 & 0.92 & 0.92 \\
\hline $\mathrm{Al}_{2} \mathrm{O}_{3}$ & 16.6 & 17.39 & 17.42 & 17.45 & 17.48 & 17.51 & 17.54 & 17.57 & 17.63 & 17.70 & 17.77 & 17.84 & 17.91 \\
\hline $\mathrm{Fe}_{2} \mathrm{O}_{3}$ & & 2.46 & 2.46 & 2.47 & 2.47 & 2.48 & 2.48 & 2.48 & 2.49 & 2.50 & 2.51 & 2.52 & 2.52 \\
\hline $\mathrm{FeO}$ & 8.22 & 5.91 & 5.91 & 5.91 & 5.91 & 5.90 & 5.90 & 5.90 & 5.89 & 5.89 & 5.88 & 5.88 & 5.87 \\
\hline $\mathrm{MnO}$ & 0.16 & 0.17 & 0.17 & 0.17 & 0.17 & 0.17 & 0.17 & 0.18 & 0.18 & 0.18 & 0.18 & 0.18 & 0.18 \\
\hline $\mathrm{MgO}$ & 6.66 & 5.71 & 5.70 & 5.69 & 5.68 & 5.67 & 5.65 & 5.64 & 5.61 & 5.59 & 5.55 & 5.52 & 5.49 \\
\hline $\mathrm{CaO}$ & 10.9 & 9.70 & 9.69 & 9.67 & 9.57 & 9.64 & 9.62 & 9.61 & 9.57 & 9.53 & 9.49 & 9.44 & 9.40 \\
\hline $\mathrm{Na}_{2} \mathrm{O}$ & 2.39 & 2.56 & 2.57 & 2.57 & 2.58 & 2.59 & 2.59 & 2.60 & 2.61 & 2.62 & 2.64 & 2.65 & 2.67 \\
\hline $\mathrm{K}_{2} \mathrm{O}$ & 1.82 & 1.96 & 1.97 & 1.97 & 1.98 & 1.98 & 1.99 & 1.99 & 2.00 & 2.02 & 2.03 & 2.04 & 2.05 \\
\hline $\mathrm{P}_{2} \mathrm{O}_{5}$ & 0.4 & 0.43 & 0.43 & 0.43 & 0.43 & 0.44 & 0.44 & 0.44 & 0.44 & 0.44 & 0.45 & 0.45 & 0.45 \\
\hline $\mathrm{H}_{2} \mathrm{O}$ & & 2.61 & 2.56 & 2.51 & 2.45 & 2.40 & 2.35 & 2.30 & 2.20 & 2.09 & 1.99 & 1.88 & 1.75 \\
\hline $\mathrm{CaO} / \mathrm{Al}_{2} \mathrm{O}_{3}$ & & 0.56 & 0.56 & 0.55 & 0.55 & 0.55 & 0.55 & 0.55 & 0.54 & 0.54 & 0.53 & 0.53 & 0.53 \\
\hline Olivine vol.\% & & 0.00 & 0.00 & 0.00 & 0.00 & 0.00 & 0.00 & 0.00 & 0.00 & 0.00 & 0.00 & 0.00 & 0.00 \\
\hline Cpx vol.\% & & 7.16 & 7.30 & 7.44 & 7.58 & 7.72 & 7.87 & 8.03 & 8.35 & 8.69 & 9.05 & 9.43 & 9.79 \\
\hline Plag vol.\% & & 0.00 & 0.00 & 0.00 & 0.00 & 0.00 & 0.00 & 0.00 & 0.00 & 0.00 & 0.00 & 0.00 & 0.00 \\
\hline Vol.\% crystals & & 7.16 & 7.30 & 7.44 & 7.58 & 7.72 & 7.87 & 8.03 & 8.35 & 8.69 & 9.05 & 9.43 & 9.79 \\
\hline
\end{tabular}

Fo\%

Bulk composition

\begin{tabular}{|c|c|c|c|c|c|c|c|c|c|c|c|c|c|}
\hline Input $\mathrm{H}_{2} \mathrm{O}$ & & 1.47 & 1.37 & 1.26 & 1.16 & 1.05 & 0.95 & 0.84 & 0.79 & 0.74 & 0.63 & 0.53 & 0.42 \\
\hline$P(\mathrm{MPa})$ & & 140 & 130 & 120 & 110 & 100 & 90 & 80 & 75 & 70 & 60 & 50 & 40 \\
\hline $\mathrm{SiO}_{2}$ & 50.9 & 50.84 & 50.93 & 51.02 & 51.11 & 51.21 & 51.32 & 51.45 & 51.52 & 51.62 & 51.84 & 52.06 & 52.29 \\
\hline $\mathrm{TiO}_{2}$ & 0.9 & 0.93 & 0.93 & 0.93 & 0.93 & 0.94 & 0.95 & 0.97 & 0.99 & 1.00 & 1.04 & 1.08 & 1.13 \\
\hline $\mathrm{Al}_{2} \mathrm{O}_{3}$ & 16.6 & 18.00 & 18.08 & 18.17 & 18.26 & 18.35 & 18.33 & 18.13 & 18.03 & 17.89 & 17.61 & 17.32 & 17.02 \\
\hline
\end{tabular}


Table 1 (continued)

\begin{tabular}{|c|c|c|c|c|c|c|c|c|c|c|c|c|c|}
\hline \multicolumn{14}{|c|}{ Bulk composition } \\
\hline $\mathrm{Fe}_{2} \mathrm{O}_{3}$ & & 2.53 & 2.53 & 2.54 & 2.54 & 2.55 & 2.57 & 2.63 & 2.66 & 2.69 & 2.76 & 2.84 & 2.92 \\
\hline $\mathrm{FeO}$ & 8.22 & 5.86 & 5.85 & 5.84 & 5.83 & 5.81 & 5.85 & 5.95 & 6.01 & 6.07 & 6.19 & 6.31 & 6.46 \\
\hline $\mathrm{MnO}$ & 0.16 & 0.18 & 0.18 & 0.18 & 0.19 & 0.19 & 0.19 & 0.20 & 0.20 & 0.20 & 0.20 & 0.21 & 0.22 \\
\hline MgO & 6.66 & 5.45 & 5.41 & 5.37 & 5.32 & 5.27 & 5.26 & 5.30 & 5.32 & 5.28 & 5.21 & 5.13 & 5.04 \\
\hline $\mathrm{CaO}$ & 10.9 & 9.34 & 9.28 & 9.22 & 9.16 & 9.09 & 8.98 & 8.82 & 8.75 & 8.69 & 8.56 & 8.43 & 8.28 \\
\hline $\mathrm{Na}_{2} \mathrm{O}$ & 2.39 & 2.69 & 2.70 & 2.72 & 2.74 & 2.76 & 2.79 & 2.81 & 2.83 & 2.84 & 2.87 & 2.90 & 2.92 \\
\hline $\mathrm{K}_{2} \mathrm{O}$ & 1.82 & 2.07 & 2.08 & 2.10 & 2.12 & 2.13 & 2.17 & 2.22 & 2.25 & 2.28 & 2.36 & 2.45 & 2.55 \\
\hline $\mathrm{P}_{2} \mathrm{O}_{5}$ & 0.4 & 0.45 & 0.46 & 0.46 & 0.46 & 0.47 & 0.48 & 0.49 & 0.50 & 0.50 & 0.52 & 0.55 & 0.57 \\
\hline $\mathrm{H}_{2} \mathrm{O}$ & & 1.67 & 1.56 & 1.45 & 1.34 & 1.23 & 1.12 & 1.03 & 0.98 & 0.93 & 0.82 & 0.72 & 0.60 \\
\hline $\mathrm{CaO} / \mathrm{Al}_{2} \mathrm{O}_{3}$ & & 0.52 & 0.51 & 0.51 & 0.50 & 0.50 & 0.49 & 0.49 & 0.49 & 0.49 & 0.49 & 0.49 & 0.49 \\
\hline Olivine vol.\% & & 0.00 & 0.00 & 0.00 & 0.00 & 0.00 & 0.00 & 0.00 & 0.03 & 0.22 & 0.61 & 1.01 & 1.41 \\
\hline Cpx vol.\% & & 10.30 & 10.70 & 11.20 & 11.70 & 12.20 & 12.70 & 13.10 & 13.20 & 13.20 & 13.30 & 13.50 & 13.70 \\
\hline Plag vol.\% & & 0.00 & 0.00 & 0.00 & 0.00 & 0.00 & 0.69 & 2.34 & 3.23 & 4.35 & 6.64 & 9.03 & 11.60 \\
\hline Vol.\% crystals & & 10.30 & 10.70 & 11.20 & 11.70 & 12.20 & 13.40 & 15.40 & 16.50 & 17.80 & 20.60 & 23.50 & 26.70 \\
\hline Fo\% & & & & & & & & & 84.00 & 84.00 & 83.80 & 83.00 & 82.80 \\
\hline An $\%$ & & & & & & & 81.80 & 80.80 & 80.80 & 79.80 & 78.80 & 77.80 & 76.50 \\
\hline \multicolumn{14}{|c|}{ Bulk composition } \\
\hline Input $\mathrm{H}_{2} \mathrm{O}$ & & & & \multicolumn{2}{|c|}{0.21} & \multicolumn{2}{|c|}{0.11} & \multicolumn{2}{|r|}{0.05} & \multicolumn{3}{|c|}{0.02} & 0.00 \\
\hline$P(\mathrm{MPa})$ & & & & \multicolumn{2}{|c|}{20} & \multicolumn{2}{|c|}{10} & \multicolumn{2}{|r|}{5} & \multicolumn{3}{|c|}{2} & 0.1 \\
\hline $\mathrm{SiO}_{2}$ & & 50.9 & & \multicolumn{2}{|c|}{52.76} & \multicolumn{2}{|c|}{52.98} & \multicolumn{2}{|r|}{53.09} & \multicolumn{3}{|c|}{53.15} & 53.19 \\
\hline $\mathrm{TiO}_{2}$ & & 0.9 & & \multicolumn{2}{|c|}{1.25} & \multicolumn{2}{|c|}{1.34} & \multicolumn{2}{|r|}{1.40} & \multicolumn{3}{|c|}{1.44} & 1.46 \\
\hline $\mathrm{Al}_{2} \mathrm{O}_{3}$ & & 16.6 & & \multicolumn{2}{|c|}{16.36} & \multicolumn{2}{|c|}{15.99} & \multicolumn{2}{|r|}{15.79} & \multicolumn{3}{|c|}{15.67} & 15.59 \\
\hline $\mathrm{Fe}_{2} \mathrm{O}_{3}$ & & & & \multicolumn{2}{|c|}{3.11} & & & & 3.28 & & 3.32 & & 3.35 \\
\hline $\mathrm{FeO}$ & & 8.22 & & & & & & & 7.12 & & 7.19 & & 7.24 \\
\hline $\mathrm{MnO}$ & & 0.16 & & & & & & & 0.24 & & 0.25 & & 0.25 \\
\hline $\mathrm{MgO}$ & & 6.66 & & & & & & & 4.60 & & 4.55 & & 4.51 \\
\hline $\mathrm{CaO}$ & & 10.9 & & & & & & & 7.67 & & 7.61 & & 7.56 \\
\hline $\mathrm{Na}_{2} \mathrm{O}$ & & 2.39 & & & & & & & 2.88 & & 2.86 & & 2.84 \\
\hline $\mathrm{K}_{2} \mathrm{O}$ & & 1.82 & & & & & & & 3.11 & & 3.19 & & 3.24 \\
\hline $\mathrm{P}_{2} \mathrm{O}_{5}$ & & 0.4 & & & & & & & 0.72 & & 0.74 & & 0.76 \\
\hline $\mathrm{H}_{2} \mathrm{O}$ & & & & & & & & & 0.09 & & 0.04 & & 0.00 \\
\hline $\mathrm{CaO} / \mathrm{Al}_{2} \mathrm{O}_{3}$ & & & & & & & & & 0.49 & & 0.49 & & 0.49 \\
\hline Olivine vol.\% & & & & & & & & & 2.92 & & 3.06 & & 3.14 \\
\hline Cpx vol.\% & & & & & & & & & 15.60 & & 15.90 & & 16.20 \\
\hline Plag vol.\% & & & & & & & & & 22.50 & & 23.70 & & 24.50 \\
\hline Vol.\% crystals & & & & & & & & & 41.00 & & 42.70 & & 43.80 \\
\hline Fo\% & & & & & & & & & 80.8 & & 79.8 & & 79.80 \\
\hline An $\%$ & & & & & & & & & 69.4 & & 68.4 & & 68.00 \\
\hline
\end{tabular}

The experiments presented by Beckett et al. (2011) show that buoyancy driven, low Reynolds number $(R e<1)$ exchange flow in a vertical pipe can form two distinct flow regimes over long time durations, representative of steady state conditions (Fig. 1): i. core annular flow (CAF) - axisymmetric flow of the two fluids where the interface between them exists at some radial distance from the centre, so that one fluid occupies the central core and the other an annulus around it. The less viscous fluid always occupies the centre. ii. side by side (SBS) flow - in which the two fluids touch the pipe walls, and the interface between them is curved and contacts the wall as two vertical lines. The flow regime formed was found to depend on viscosity ratio $(\beta)$, defined as the viscosity of the more viscous fluid divided by the viscosity of the less viscous fluid. CAF occurred at $\beta \gtrsim 100$ and SBS flow at $\beta \lesssim 100$. A subset of the SBS flows, termed transitional side by side (TSBS) flow, were observed at $\beta=5-59$. In TSBS flow both CAF and SBS were observed simultaneously within different parts of the pipe; the majority of the vertical extent of the flow was SBS (and was found to increase in length with time during three experiments), but at some height in the upper portion of the pipe the ascending fluid moved off the pipe wall forming a core annular flow. Obtained velocity profiles of the CAF and SBS flows show that at the fluid interface, a portion of the less dense, less viscous fluid is dragged downwards by the more viscous fluid i.e. a portion of the less dense fluid has a negative velocity (Fig. 1). The volume flux (Q) of an exchange flow is defined as the net ascending flux of the less dense, less viscous fluid, which in the analogue experiments is constrained to be equal to the net downwards flux of the more dense, more viscous fluid. The experiments show that exchange flows do not adopt a flow regime that maximises volume flux. Instead it is shown that the volume flux of the flow does not depend on the flow regime and is given by the following empirical relationship based on dimensional scaling for volume flux $(Q)$ :

$Q=\left(0.059 \beta^{-0.74}\right) \frac{g \Delta \rho r_{2}^{4}}{2 \mu_{1}}$,

where $g$ is gravitational acceleration, $\Delta \rho$ is density difference, $r_{2}$ is the pipe radius and $\mu_{1}$ is the viscosity of the less viscous fluid.

It is worth noting that Eq. (1) is the only dimensional form for expressing the exchange flow flux in terms of the properties of the fluids and conduit. Other studies have also identified this form (Kazahaya et al., 1994; Stevenson and Blake, 1998; Huppert and Hallworth, 2007; Kerswell, 2011; Palma et al., 2011), with different choices of the rising or descending magma viscosities and different coefficients to account for non-maximal exchange flow occurring. In particular, Palma et al. (2011), Kerswell (2011) and Beckett et al. (2011) presented detailed consideration of the system parameter space and its relationship to the condition of maximal volumetric flux of the exchange flow. The primary conclusion of these studies is that laboratory and natural vertical exchange flow of two fluids does not occur at maximal flux, and there is some relationship between the exchange flux and flow pattern adopted. Currently, there is incomplete understanding of the relationship between the exchange flux and the pressure gradients in a vertical region containing two fluids of different densities, and this restricts progress in volcanological applications to the use of empirical relationships such as Eq. (1) or flux coefficient values identified by Huppert and Hallworth (2007) and Palma et al. (2011). 
Table 2

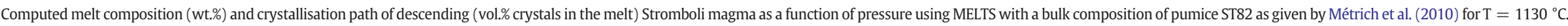

\begin{tabular}{|c|c|c|c|c|c|c|c|c|c|c|c|c|c|}
\hline & Bulk composition & MELTS & & & & & & & & & & & \\
\hline Input $\mathrm{H}_{2} \mathrm{O}$ & & 0.0011 & 0.0011 & 0.0011 & 0.0011 & 0.0011 & 0.0011 & 0.0011 & 0.0011 & 0.0011 & 0.0011 & 0.0011 & 0.0011 \\
\hline$P(\mathrm{MPa})$ & & 350 & 345 & 340 & 335 & 330 & 325 & 320 & 315 & 310 & 305 & 300 & 295 \\
\hline $\mathrm{SiO}_{2}$ & 50.9 & 53.49 & 53.49 & 53.48 & 53.47 & 53.47 & 53.46 & 53.45 & 53.44 & 53.43 & 53.42 & 53.41 & 53.40 \\
\hline $\mathrm{TiO}_{2}$ & 0.9 & 2.14 & 2.13 & 2.12 & 2.11 & 2.10 & 2.09 & 2.08 & 2.06 & 2.05 & 2.04 & 2.03 & 2.02 \\
\hline $\mathrm{Al}_{2} \mathrm{O}_{3}$ & 16.6 & 17.99 & 17.96 & 17.94 & 17.91 & 17.88 & 17.85 & 17.83 & 17.80 & 17.77 & 17.74 & 17.71 & 17.68 \\
\hline $\mathrm{Fe}_{2} \mathrm{O}_{3}$ & & 2.13 & 2.15 & 2.18 & 2.20 & 2.22 & 2.24 & 2.26 & 2.28 & 2.30 & 2.32 & 2.34 & 2.36 \\
\hline $\mathrm{FeO}$ & 8.22 & 5.04 & 5.08 & 5.13 & 5.17 & 5.21 & 5.25 & 5.30 & 5.34 & 5.38 & 5.43 & 5.47 & 5.52 \\
\hline $\mathrm{MnO}$ & 0.16 & 0.64 & 0.63 & 0.63 & 0.62 & 0.61 & 0.61 & 0.60 & 0.60 & 0.59 & 0.58 & 0.58 & 0.57 \\
\hline $\mathrm{MgO}$ & 6.66 & 2.33 & 2.35 & 2.37 & 2.40 & 2.42 & 2.44 & 2.47 & 2.49 & 2.52 & 2.54 & 2.57 & 2.59 \\
\hline $\mathrm{CaO}$ & 10.9 & 6.34 & 6.34 & 6.34 & 6.34 & 6.34 & 6.35 & 6.35 & 6.35 & 6.36 & 6.36 & 6.36 & 6.37 \\
\hline $\mathrm{Na}_{2} \mathrm{O}$ & 2.39 & 2.57 & 2.59 & 2.60 & 2.62 & 2.63 & 2.65 & 2.66 & 2.68 & 2.69 & 2.71 & 2.72 & 2.73 \\
\hline $\mathrm{K}_{2} \mathrm{O}$ & 1.82 & 5.72 & 5.68 & 5.65 & 5.61 & 5.58 & 5.54 & 5.03 & 5.47 & 5.43 & 5.39 & 5.36 & 5.32 \\
\hline $\mathrm{P}_{2} \mathrm{O}_{5}$ & 0.4 & 1.60 & 1.58 & 1.57 & 1.55 & 1.54 & 1.52 & 1.50 & 1.49 & 1.47 & 1.46 & 1.44 & 1.43 \\
\hline $\mathrm{H}_{2} \mathrm{O}$ & & 0.00 & 0.00 & 0.00 & 0.00 & 0.00 & 0.00 & 0.00 & 0.00 & 0.00 & 0.00 & 0.00 & 0.00 \\
\hline $\begin{array}{l}\mathrm{CaO} / \mathrm{Al}_{2} \mathrm{O}_{3} \\
\text { Olivine vol.\% }\end{array}$ & & 0.35 & 0.35 & 0.35 & 0.35 & 0.36 & 0.36 & 0.36 & 0.36 & 0.36 & 0.36 & 0.36 & 0.36 \\
\hline Cpx vol.\% & & 36.30 & 36.20 & 36.00 & 35.90 & 35.70 & 35.60 & 35.40 & 35.30 & 35.10 & 35.00 & 34.80 & 34.70 \\
\hline Plag vol.\% & & 35.80 & 35.70 & 35.50 & 35.40 & 35.30 & 35.10 & 35.00 & 34.80 & 34.70 & 34.50 & 34.40 & 34.20 \\
\hline Vol.\% crystals & & 72.10 & 71.80 & 71.50 & 71.30 & 71.00 & 70.70 & 70.40 & 70.10 & 69.80 & 69.50 & 69.20 & 68.90 \\
\hline Fo\% & & & & & & & & & & & & & \\
\hline \multirow[t]{2}{*}{ An\% } & & 55.30 & 55.90 & 55.90 & 55.90 & 55.90 & 56.40 & 56.40 & 56.40 & 56.40 & 56.40 & 56.40 & 57.40 \\
\hline & Bulk composition & MELTS & & & & & & & & & & & \\
\hline Input $\mathrm{H}_{2} \mathrm{O}$ & & 0.0011 & 0.0011 & 0.0011 & 0.0011 & 0.0011 & 0.0011 & 0.0011 & 0.0011 & 0.0011 & 0.0011 & 0.0011 & 0.0011 \\
\hline$P(\mathrm{MPa})$ & & 290 & 285 & 280 & 275 & 270 & 265 & 260 & 255 & 250 & 245 & 240 & 235 \\
\hline $\mathrm{SiO}_{2}$ & 50.9 & 53.39 & 53.38 & 53.36 & 53.35 & 53.34 & 53.32 & 53.31 & 53.29 & 53.28 & 53.26 & 53.24 & 53.23 \\
\hline $\mathrm{TiO}_{2}$ & 0.9 & 2.01 & 2.00 & 1.99 & 1.98 & 1.97 & 1.96 & 1.95 & 1.94 & 1.93 & 1.92 & 1.91 & 1.90 \\
\hline $\mathrm{Al}_{2} \mathrm{O}_{3}$ & 16.6 & 17.66 & 17.63 & 17.60 & 17.57 & 17.54 & 17.51 & 17.48 & 17.45 & 17.42 & 17.39 & 17.36 & 17.33 \\
\hline $\mathrm{Fe}_{2} \mathrm{O}_{3}$ & & 2.38 & 2.40 & 2.42 & 2.44 & 2.46 & 2.49 & 2.51 & 2.53 & 2.55 & 2.57 & 2.59 & 2.61 \\
\hline $\mathrm{FeO}$ & 8.22 & 5.56 & 5.60 & 5.65 & 5.69 & 5.73 & 5.78 & 5.82 & 5.86 & 5.91 & 5.95 & 5.99 & 6.03 \\
\hline $\mathrm{MnO}$ & 0.16 & 0.57 & 0.56 & 0.55 & 0.55 & 0.54 & 0.54 & 0.53 & 0.53 & 0.52 & 0.51 & 0.51 & 0.50 \\
\hline MgO & 6.66 & 2.62 & 2.65 & 2.67 & 2.70 & 2.73 & 2.76 & 2.79 & 2.82 & 2.85 & 2.88 & 2.91 & 2.94 \\
\hline $\mathrm{CaO}$ & 10.9 & 6.37 & 6.38 & 6.39 & 6.39 & 6.40 & 6.41 & 6.41 & 6.42 & 6.43 & 6.44 & 6.45 & 6.46 \\
\hline $\mathrm{Na}_{2} \mathrm{O}$ & 2.39 & 2.75 & 2.76 & 2.77 & 2.78 & 2.79 & 2.81 & 2.82 & 2.83 & 2.84 & 2.85 & 2.86 & 2.87 \\
\hline $\mathrm{K}_{2} \mathrm{O}$ & 1.82 & 5.28 & 5.24 & 5.21 & 5.17 & 5.13 & 5.09 & 5.06 & 5.02 & 4.98 & 4.94 & 4.91 & 4.87 \\
\hline $\mathrm{P}_{2} \mathrm{O}_{5}$ & 0.4 & 1.41 & 1.40 & 1.38 & 1.37 & 1.35 & 1.34 & 1.33 & 1.31 & 1.30 & 1.28 & 1.27 & 1.26 \\
\hline $\mathrm{H}_{2} \mathrm{O}$ & & 0.00 & 0.00 & 0.00 & 0.00 & 0.00 & 0.00 & 0.00 & 0.00 & 0.00 & 0.00 & 0.00 & 0.00 \\
\hline $\begin{array}{l}\mathrm{CaO} / \mathrm{Al}_{2} \mathrm{O}_{3} \\
\text { Olivine vol.\% }\end{array}$ & & 0.36 & 0.36 & 0.36 & 0.36 & 0.37 & 0.37 & 0.37 & 0.37 & 0.37 & 0.37 & 0.37 & 0.37 \\
\hline Cpx vol.\% & & 34.50 & 34.30 & 34.20 & 34.00 & 33.80 & 33.60 & 33.50 & 33.30 & 33.10 & 32.90 & 32.70 & 32.50 \\
\hline Plag vol.\% & & 34.10 & 33.90 & 33.80 & 33.60 & 33.50 & 33.30 & 33.10 & 33.00 & 32.80 & 32.70 & 32.50 & 32.30 \\
\hline Vol.\% crystals & & 68.60 & 68.30 & 67.90 & 67.60 & 67.30 & 66.90 & 66.60 & 66.30 & 65.90 & 65.60 & 65.20 & 64.90 \\
\hline \multirow{3}{*}{$\begin{array}{l}\text { Fo\% } \\
\text { An\% }\end{array}$} & & & & & & & & & & & & & \\
\hline & & 57.40 & 57.40 & 57.40 & 57.40 & 57.90 & 57.90 & 57.90 & 58.50 & 58.50 & 58.90 & 58.90 & 58.90 \\
\hline & Bulk composition & MELTS & & & & & & & & & & & \\
\hline Input $\mathrm{H}_{2} \mathrm{O}$ & & 0.0011 & 0.0011 & 0.0011 & 0.0011 & 0.0011 & 0.0011 & 0.0011 & 0.0011 & 0.0011 & 0.0011 & 0.0011 & 0.0011 \\
\hline$P(\mathrm{MPa})$ & & 230 & 225 & 220 & 215 & 210 & 205 & 200 & 190 & 180 & 170 & 160 & 150 \\
\hline $\mathrm{SiO}_{2}$ & 50.9 & 53.21 & 53.19 & 53.18 & 53.16 & 53.14 & 53.12 & 53.10 & 53.06 & 53.02 & 52.98 & 52.94 & 52.90 \\
\hline $\mathrm{TiO}_{2}$ & 0.9 & 1.89 & 1.88 & 1.87 & 1.86 & 1.85 & 1.84 & 1.83 & 1.81 & 1.79 & 1.77 & 1.76 & 1.74 \\
\hline $\mathrm{Al}_{2} \mathrm{O}_{3}$ & 16.6 & 17.30 & 17.27 & 17.24 & 17.21 & 17.18 & 17.15 & 17.12 & 17.06 & 17.00 & 16.94 & 16.88 & 16.82 \\
\hline $\mathrm{Fe}_{2} \mathrm{O}_{3}$ & & 2.63 & 2.65 & 2.67 & 2.69 & 2.71 & 2.73 & 2.75 & 2.79 & 2.83 & 2.87 & 2.90 & 2.94 \\
\hline $\mathrm{FeO}$ & 8.22 & 6.08 & 6.12 & 6.16 & 6.20 & 6.24 & 6.28 & 6.32 & 6.40 & 6.47 & 6.55 & 6.62 & 6.69 \\
\hline $\mathrm{MnO}$ & 0.16 & 0.48 & 0.49 & 0.49 & 0.48 & 0.48 & 0.47 & 0.47 & 0.46 & 0.45 & 0.44 & 0.43 & 0.42 \\
\hline $\mathrm{MgO}$ & 6.66 & 2.97 & 3.00 & 3.03 & 3.07 & 3.10 & 3.13 & 3.17 & 3.24 & 3.31 & 3.38 & 3.46 & 3.54 \\
\hline
\end{tabular}




\begin{tabular}{|c|c|c|c|c|c|c|c|c|c|c|c|c|c|}
\hline $\mathrm{CaO}$ & 10.9 & 6.47 & 6.48 & 6.49 & 6.50 & 6.51 & 6.53 & 6.58 & 6.57 & 6.59 & 6.62 & 6.66 & 6.69 \\
\hline $\mathrm{Na}_{2} \mathrm{O}$ & 2.39 & 2.88 & 2.89 & 2.90 & 2.90 & 2.91 & 2.92 & 2.93 & 2.94 & 2.95 & 2.96 & 2.97 & 2.98 \\
\hline $\mathrm{K}_{2} \mathrm{O}$ & 1.82 & 4.83 & 4.79 & 4.76 & 4.72 & 4.68 & 4.64 & 4.61 & 4.53 & 4.46 & 4.39 & 4.32 & 4.25 \\
\hline $\mathrm{P}_{2} \mathrm{O}_{5}$ & 0.4 & 1.24 & 1.23 & 1.22 & 1.21 & 1.19 & 1.18 & 1.17 & 1.14 & 1.12 & 1.09 & 1.07 & 1.05 \\
\hline $\mathrm{H}_{2} \mathrm{O}$ & & 0.00 & 0.00 & 0.00 & 0.00 & 0.00 & 0.00 & 0.00 & 0.00 & 0.00 & 0.00 & 0.00 & 0.00 \\
\hline \multicolumn{14}{|l|}{$\begin{array}{l}\text { Olivine vol. } \\
\text { Olint }\end{array}$} \\
\hline Cpx vol.\% & & 32.30 & 32.10 & 31.90 & 31.70 & 31.50 & 31.30 & 31.10 & 30.70 & 30.30 & 29.80 & 29.40 & 28.90 \\
\hline Plag vol.\% & & 32.20 & 32.00 & 31.80 & 31.70 & 31.50 & 31.30 & 31.20 & 30.80 & 30.50 & 30.10 & 29.80 & 29.40 \\
\hline Vol.\% crystals & & 64.50 & 64.10 & 63.80 & 63.40 & 63.00 & 62.70 & 62.30 & 61.50 & 60.70 & 59.90 & 59.10 & 58.30 \\
\hline \multicolumn{14}{|l|}{ Fo\% } \\
\hline \multirow[t]{2}{*}{ An\% } & & 58.90 & 58.90 & 60.00 & 60.00 & 60.00 & 60.00 & 60.00 & 60.40 & 61.10 & 61.50 & 61.50 & 62.50 \\
\hline & Bulk composition & MELTS & & & & & & & & & & & \\
\hline Input $\mathrm{H}_{2} \mathrm{O}$ & & 0.0011 & 0.0011 & 0.0011 & 0.0011 & 0.0011 & 0.0011 & 0.0011 & 0.0011 & 0.0011 & 0.0011 & 0.0011 & 0.0011 \\
\hline$P(\mathrm{MPa})$ & & 140 & 130 & 120 & 110 & 100 & 90 & 80 & 75 & 70 & 60 & 50 & 40 \\
\hline $\mathrm{SiO}_{2}$ & 50.9 & 52.85 & 52.81 & 52.77 & 52.82 & 52.88 & 52.94 & 53.01 & 53.04 & 53.08 & 53.15 & 53.22 & 53.22 \\
\hline $\mathrm{TiO}_{2}$ & 0.9 & 1.72 & 1.70 & 1.68 & 1.66 & 1.65 & 1.63 & 1.61 & 1.60 & 1.59 & 1.56 & 1.54 & 1.53 \\
\hline $\mathrm{Al}_{2} \mathrm{O}_{3}$ & 16.6 & 16.76 & 16.70 & 16.64 & 16.54 & 16.44 & 16.33 & 16.23 & 16.18 & 16.12 & 16.02 & 15.92 & 15.85 \\
\hline $\mathrm{Fe}_{2} \mathrm{O}_{3}$ & & 2.98 & 3.01 & 3.04 & 3.08 & 3.11 & 3.14 & 3.17 & 3.18 & 3.19 & 3.22 & 3.25 & 3.27 \\
\hline $\mathrm{FeO}$ & 8.22 & 6.75 & 6.81 & 6.87 & 6.93 & 6.99 & 7.04 & 7.10 & 7.12 & 7.14 & 7.19 & 7.22 & 7.23 \\
\hline $\mathrm{MnO}$ & 0.16 & 0.41 & 0.40 & 0.39 & 0.37 & 0.35 & 0.33 & 0.32 & 0.31 & 0.30 & 0.28 & 0.27 & 0.26 \\
\hline MgO & 6.66 & 3.62 & 3.70 & 3.78 & 3.84 & 3.90 & 3.97 & 4.03 & 4.07 & 4.10 & 4.16 & 4.23 & 4.29 \\
\hline $\mathrm{CaO}$ & 10.9 & 6.72 & 6.76 & 6.80 & 6.85 & 6.90 & 6.95 & 7.01 & 7.04 & 7.07 & 7.13 & 7.19 & 7.26 \\
\hline $\mathrm{Na}_{2} \mathrm{O}$ & 2.39 & 2.99 & 2.99 & 2.99 & 2.98 & 2.96 & 2.94 & 2.92 & 2.91 & 2.90 & 2.88 & 2.86 & 2.85 \\
\hline $\mathrm{K}_{2} \mathrm{O}$ & 1.82 & 4.18 & 4.11 & 4.04 & 3.96 & 3.88 & 3.80 & 3.72 & 3.68 & 3.64 & 3.55 & 3.47 & 3.42 \\
\hline $\mathrm{P}_{2} \mathrm{O}_{5}$ & 0.4 & 1.03 & 1.01 & 0.98 & 0.96 & 0.94 & 0.92 & 0.89 & 0.88 & 0.87 & 0.85 & 0.82 & 0.81 \\
\hline $\mathrm{H}_{2} \mathrm{O}$ & & 0.00 & 0.00 & 0.00 & 0.00 & 0.00 & 0.00 & 0.00 & 0.00 & 0.00 & 0.00 & 0.00 & 0.00 \\
\hline $\mathrm{CaO} / \mathrm{Al}_{2} \mathrm{O}_{3}$ & & 0.40 & 0.41 & 0.41 & 0.41 & 0.42 & 0.43 & 0.43 & 0.44 & 0.44 & 0.45 & 0.45 & 0.46 \\
\hline Olivine vol.\% & & 0.31 & 0.67 & 1.05 & 1.46 & 1.68 & 1.90 & 2.36 & 2.78 & 2.89 & & & \\
\hline Cpx vol.\% & & 28.40 & 27.90 & 27.40 & 26.50 & 25.40 & 24.20 & 23.00 & 22.40 & 21.80 & 20.40 & 19.20 & 18.50 \\
\hline Plag vol.\% & & 29.00 & 28.70 & 28.30 & 28.00 & 27.70 & 27.40 & 27.00 & 26.90 & 26.70 & 26.30 & 25.90 & 25.60 \\
\hline Vol.\% crystals & & 57.50 & 56.60 & 55.70 & 54.80 & 53.70 & 52.70 & 51.50 & 51.00 & 50.40 & 49.10 & 47.90 & 47.10 \\
\hline Fo\% & & & & & & & & & 78.60 & 78.80 & 78.80 & 78.80 & 78.80 \\
\hline \multirow[t]{2}{*}{ An\% } & & 62.50 & 62.50 & 63.50 & 63.50 & 63.90 & 64.60 & 64.90 & 64.90 & 64.90 & 65.60 & 66.00 & 66.00 \\
\hline & & Bulk composition & & & MELTS & & & & & & & & \\
\hline Input $\mathrm{H}_{2} \mathrm{O}$ & & & & & & \multicolumn{2}{|c|}{0.0011} & \multicolumn{2}{|r|}{0.0011} & \multicolumn{3}{|c|}{0.0011} & 0.0011 \\
\hline$P(\mathrm{MPa})$ & & & & \multicolumn{2}{|c|}{20} & \multicolumn{2}{|c|}{10} & \multirow{2}{*}{\multicolumn{2}{|c|}{5}} & \multicolumn{3}{|c|}{2} & 0.1 \\
\hline $\mathrm{SiO}_{2}$ & & 50.9 & & \multicolumn{2}{|c|}{53.21} & \multicolumn{2}{|c|}{53.20} & & 53.20 & & 53.19 & & 53.19 \\
\hline $\mathrm{TiO}_{2}$ & & 0.9 & & & & & & & 1.47 & & 1.47 & & 1.46 \\
\hline $\mathrm{Al}_{2} \mathrm{O}_{3}$ & & 16.6 & & & & & & & 15.62 & & 15.60 & & 15.59 \\
\hline $\mathrm{Fe}_{2} \mathrm{O}_{3}$ & & & & & & & & & 3.34 & & 3.34 & & 3.35 \\
\hline $\mathrm{FeO}$ & & 8.22 & & & & & & & 7.24 & & 7.24 & & 7.24 \\
\hline MnO & & 0.16 & & & & & & & 0.25 & & 0.25 & & 0.25 \\
\hline MgO & & 6.66 & & & & & & & 4.49 & & 4.50 & & 4.51 \\
\hline $\mathrm{CaO}$ & & 10.9 & & & & & & & 7.53 & & 7.55 & & 7.56 \\
\hline $\mathrm{Na}_{2} \mathrm{O}$ & & 2.39 & & & & & & & 2.84 & & 2.84 & & 2.84 \\
\hline $\mathrm{K}_{2} \mathrm{O}$ & & 1.82 & & & & & & & 3.22 & & 3.25 & & 3.24 \\
\hline $\mathrm{P}_{2} \mathrm{O}_{5}$ & & 0.4 & & & & & & & 0.76 & & 0.76 & & 0.76 \\
\hline $\mathrm{H}_{2} \mathrm{O}$ & & & & & & & & & 0.00 & & 0.00 & & 0.00 \\
\hline $\mathrm{CaO} / \mathrm{Al}_{2} \mathrm{O}_{3}$ & & & & & & & & & 0.48 & & 0.48 & & 0.49 \\
\hline Olivine vol.\% & & & & & & & & & 3.12 & & 3.13 & & 3.14 \\
\hline Cpx vol.\% & & & & & & & & & 16.50 & & 16.30 & & 16.20 \\
\hline Plag vol.\% & & & & & & & & & 24.60 & & 24.50 & & 24.50 \\
\hline Vol.\% crystals & & & & & & & & & 44.20 & & 43.90 & & 43.80 \\
\hline Fo\% & & & & & & & & & 79.80 & & 79.80 & & 79.80 \\
\hline An\% & & & & & & & & & 68.00 & & 68.00 & & 68.00 \\
\hline
\end{tabular}


Table 3

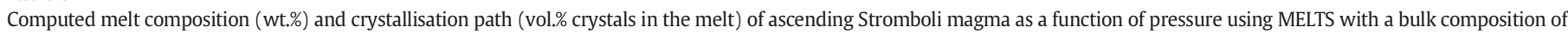
pumice ST82 as given by Métrich et al. (2010) for T $=1115{ }^{\circ} \mathrm{C}$.

\begin{tabular}{|c|c|c|c|c|c|c|c|c|c|c|c|c|c|}
\hline \multicolumn{14}{|c|}{ Bulk composition } \\
\hline Input $\mathrm{H}_{2} \mathrm{O}$ & & 3.64 & 3.59 & 3.54 & 3.48 & 3.43 & 3.38 & 3.33 & 3.28 & 3.22 & 3.17 & 3.12 & 3.07 \\
\hline$P(\mathrm{MPa})$ & & 350 & 345 & 340 & 335 & 330 & 325 & 320 & 315 & 310 & 305 & 300 & 295 \\
\hline $\mathrm{SiO}_{2}$ & 50.9 & 49.57 & 49.60 & 49.63 & 49.66 & 49.69 & 49.72 & 49.75 & 49.79 & 49.82 & 49.85 & 49.88 & 49.91 \\
\hline $\mathrm{TiO}_{2}$ & 0.9 & 0.90 & 0.88 & 0.88 & 0.88 & 0.88 & 0.88 & 0.88 & 0.88 & 0.89 & 0.89 & 0.89 & 0.89 \\
\hline $\mathrm{Al}_{2} \mathrm{O}_{3}$ & 16.6 & 17.59 & 17.60 & 17.62 & 17.64 & 17.66 & 17.68 & 17.70 & 17.72 & 17.73 & 17.75 & 17.78 & 17.80 \\
\hline $\mathrm{Fe}_{2} \mathrm{O}_{3}$ & & 2.31 & 2.32 & 2.32 & 2.33 & 2.33 & 2.34 & 2.34 & 2.35 & 2.35 & 2.36 & 2.36 & 2.37 \\
\hline $\mathrm{FeO}$ & 8.22 & 5.93 & 5.93 & 5.93 & 5.93 & 5.93 & 5.92 & 5.92 & 5.92 & 5.92 & 5.92 & 5.92 & 5.92 \\
\hline $\mathrm{MnO}$ & 0.16 & 0.18 & 0.18 & 0.18 & 0.18 & 0.18 & 0.18 & 0.18 & 0.18 & 0.18 & 0.18 & 0.18 & 0.18 \\
\hline $\mathrm{MgO}$ & 6.66 & 5.22 & 5.22 & 5.22 & 5.22 & 5.22 & 5.22 & 5.22 & 5.22 & 5.22 & 5.22 & 5.21 & 5.21 \\
\hline $\mathrm{CaO}$ & 10.9 & 9.25 & 9.25 & 9.24 & 9.23 & 9.22 & 9.22 & 9.21 & 9.20 & 9.19 & 9.18 & 9.17 & 9.16 \\
\hline $\mathrm{Na}_{2} \mathrm{O}$ & 2.39 & 2.61 & 2.61 & 2.62 & 2.62 & 2.62 & 2.63 & 2.63 & 2.63 & 2.64 & 2.64 & 2.65 & 2.65 \\
\hline $\mathrm{K}_{2} \mathrm{O}$ & 1.82 & 2.00 & 2.01 & 2.01 & 2.01 & 2.02 & 2.02 & 2.02 & 2.03 & 2.03 & 2.03 & 2.04 & 2.04 \\
\hline $\mathrm{P}_{2} \mathrm{O}_{5}$ & 0.4 & 0.44 & 0.44 & 0.44 & 0.44 & 0.44 & 0.44 & 0.44 & 0.45 & 0.45 & 0.45 & 0.45 & 0.45 \\
\hline $\mathrm{H}_{2} \mathrm{O}$ & & 4.01 & 3.96 & 3.91 & 3.85 & 3.80 & 3.75 & 3.70 & 3.65 & 3.59 & 3.54 & 3.49 & 3.44 \\
\hline $\mathrm{CaO} / \mathrm{Al}_{2} \mathrm{O}_{3}$ & & 0.53 & 0.53 & 0.52 & 0.52 & 0.52 & 0.52 & 0.52 & 0.52 & 0.52 & 0.52 & 0.52 & 0.51 \\
\hline Olivine vol.\% & & 0.23 & 0.22 & 0.21 & 0.20 & 0.20 & 0.19 & 0.18 & 0.17 & 0.16 & 0.15 & 0.14 & 0.13 \\
\hline Cpx vol.\% & & 9.32 & 9.38 & 9.47 & 9.54 & 9.61 & 9.69 & 9.78 & 9.87 & 9.95 & 10.00 & 10.10 & 10.20 \\
\hline Plag vol.\% & & 0.00 & 0.00 & 0.00 & 0.00 & 0.00 & 0.00 & 0.00 & 0.00 & 0.00 & 0.00 & 0.00 & 0.00 \\
\hline Vol.\% crystals & & 9.55 & 9.60 & 9.68 & 9.74 & 9.81 & 9.88 & 9.96 & 10.00 & 10.10 & 10.20 & 10.30 & 10.40 \\
\hline Fo\% & & 84.80 & 84.80 & 84.80 & 84.80 & 84.80 & 84.80 & 84.80 & 84.80 & 84.80 & 84.80 & 84.80 & 84.80 \\
\hline
\end{tabular}

An\%

Bulk composition

\begin{tabular}{|c|c|c|c|c|c|c|c|c|c|c|c|c|c|}
\hline Input $\mathrm{H}_{2} \mathrm{O}$ & & 3.02 & 2.96 & 2.91 & 2.86 & 2.81 & 2.76 & 2.70 & 2.65 & 2.60 & 2.55 & 2.50 & 2.44 \\
\hline$P(\mathrm{MPa})$ & & 290 & 285 & 280 & 275 & 270 & 265 & 260 & 255 & 250 & 245 & 240 & 235 \\
\hline $\mathrm{SiO}_{2}$ & 50.9 & 49.94 & 49.97 & 50.01 & 50.04 & 50.07 & 50.10 & 50.14 & 50.17 & 50.20 & 50.24 & 50.27 & 50.31 \\
\hline $\mathrm{TiO}_{2}$ & 0.9 & 0.89 & 0.89 & 0.89 & 0.89 & 0.89 & 0.89 & 0.89 & 0.89 & 0.90 & 0.90 & 0.90 & 0.90 \\
\hline $\mathrm{Al}_{2} \mathrm{O}_{3}$ & 16.6 & 17.82 & 17.84 & 17.86 & 17.88 & 17.91 & 17.93 & 17.95 & 17.98 & 18.00 & 18.03 & 18.05 & 18.08 \\
\hline $\mathrm{Fe}_{2} \mathrm{O}_{3}$ & & 2.37 & 2.37 & 2.38 & 2.38 & 2.39 & 2.39 & 2.40 & 2.40 & 2.41 & 2.41 & 2.41 & 2.42 \\
\hline $\mathrm{FeO}$ & 8.22 & 5.91 & 5.91 & 5.91 & 5.91 & 5.91 & 5.90 & 5.90 & 5.90 & 5.90 & 5.89 & 5.89 & 5.89 \\
\hline $\mathrm{MnO}$ & 0.16 & 0.18 & 0.18 & 0.18 & 0.18 & 0.18 & 0.18 & 0.18 & 0.18 & 0.18 & 0.18 & 0.18 & 0.18 \\
\hline $\mathrm{MgO}$ & 6.66 & 5.21 & 5.21 & 5.20 & 5.20 & 5.20 & 5.19 & 5.19 & 5.19 & 5.18 & 5.18 & 5.17 & 5.17 \\
\hline $\mathrm{CaO}$ & 10.9 & 9.15 & 9.14 & 9.13 & 9.12 & 9.10 & 9.09 & 9.08 & 9.06 & 9.05 & 9.03 & 9.02 & 9.00 \\
\hline $\mathrm{Na}_{2} \mathrm{O}$ & 2.39 & 2.65 & 2.66 & 2.66 & 2.67 & 2.67 & 2.68 & 2.68 & 2.69 & 2.69 & 2.70 & 2.70 & 2.71 \\
\hline $\mathrm{K}_{2} \mathrm{O}$ & 1.82 & 2.04 & 2.05 & 2.05 & 2.05 & 2.06 & 2.06 & 2.07 & 2.07 & 2.07 & 2.08 & 2.08 & 2.09 \\
\hline $\mathrm{P}_{2} \mathrm{O}_{5}$ & 0.4 & 0.45 & 0.45 & 0.45 & 0.45 & 0.45 & 0.45 & 0.45 & 0.45 & 0.46 & 0.46 & 0.46 & 0.46 \\
\hline $\mathrm{H}_{2} \mathrm{O}$ & & 3.38 & 3.33 & 3.28 & 3.23 & 3.17 & 3.12 & 3.07 & 3.02 & 2.96 & 2.91 & 2.86 & 2.80 \\
\hline $\mathrm{CaO} / \mathrm{Al}_{2} \mathrm{O}_{3}$ & & 0.51 & 0.51 & 0.51 & 0.51 & 0.51 & 0.51 & 0.51 & 0.50 & 0.50 & 0.50 & 0.50 & 0.50 \\
\hline Olivine vol.\% & & 0.12 & 0.12 & 0.11 & 0.10 & 0.08 & 0.07 & 0.07 & 0.06 & 0.05 & 0.03 & 0.03 & 0.02 \\
\hline Cpx vol.\% & & 10.30 & 10.40 & 10.50 & 10.60 & 10.80 & 10.90 & 11.00 & 11.10 & 11.20 & 11.40 & 11.50 & 11.60 \\
\hline Plag vol.\% & & 0.00 & 0.00 & 0.00 & 0.00 & 0.00 & 0.00 & 0.00 & 0.00 & 0.00 & 0.00 & 0.00 & 0.00 \\
\hline Vol.\% crystals & & 10.50 & 10.60 & 10.60 & 10.70 & 10.80 & 10.90 & 11.10 & 11.20 & 11.30 & 11.40 & 11.50 & 11.60 \\
\hline Fo $\%$ & & 84.80 & 84.80 & 84.80 & 84.00 & 84.00 & 84.00 & 84.00 & 84.00 & 84.00 & 84.00 & 84.00 & 84.00 \\
\hline
\end{tabular}

An\%

Bulk composition

\begin{tabular}{|c|c|c|c|c|c|c|c|c|c|c|c|c|c|}
\hline Input $\mathrm{H}_{2} \mathrm{O}$ & & 2.39 & 2.34 & 2.29 & 2.24 & 2.18 & 2.13 & 2.08 & 1.98 & 1.87 & 1.77 & 1.66 & 1.56 \\
\hline$P(\mathrm{MPa})$ & & 230 & 225 & 220 & 215 & 210 & 205 & 200 & 190 & 180 & 170 & 160 & 150 \\
\hline $\mathrm{SiO}_{2}$ & 50.9 & 50.34 & 50.38 & 50.42 & 50.46 & 50.50 & 50.54 & 50.58 & 50.66 & 50.74 & 50.83 & 50.92 & 51.01 \\
\hline $\mathrm{TiO}_{2}$ & 0.9 & 0.90 & 0.90 & 0.90 & 0.90 & 0.90 & 0.90 & 0.90 & 0.91 & 0.91 & 0.91 & 0.91 & 0.91 \\
\hline $\mathrm{Al}_{2} \mathrm{O}_{3}$ & 16.6 & 18.10 & 18.13 & 18.16 & 18.19 & 18.22 & 18.25 & 18.28 & 18.34 & 18.41 & 18.48 & 18.55 & 18.63 \\
\hline $\mathrm{Fe}_{2} \mathrm{O}_{3}$ & & 2.42 & 2.43 & 2.43 & 2.43 & 2.44 & 2.44 & 2.44 & 2.45 & 2.45 & 2.46 & 2.46 & 2.47 \\
\hline $\mathrm{FeO}$ & 8.22 & 5.89 & 5.88 & 5.88 & 5.87 & 5.87 & 5.87 & 5.86 & 5.85 & 5.84 & 5.83 & 5.81 & 5.80 \\
\hline $\mathrm{MnO}$ & 0.16 & 0.18 & 0.18 & 0.18 & 0.19 & 0.19 & 0.19 & 0.19 & 0.19 & 0.19 & 0.19 & 0.19 & 0.19 \\
\hline $\mathrm{MgO}$ & 6.66 & 5.16 & 5.15 & 5.14 & 5.13 & 5.12 & 5.11 & 5.10 & 5.07 & 5.04 & 5.01 & 4.98 & 4.95 \\
\hline $\mathrm{CaO}$ & 10.9 & 8.99 & 8.97 & 8.95 & 8.94 & 8.92 & 8.90 & 8.89 & 8.85 & 8.81 & 8.76 & 8.71 & 8.66 \\
\hline $\mathrm{Na}_{2} \mathrm{O}$ & 2.39 & 2.71 & 2.72 & 2.73 & 2.73 & 2.74 & 2.74 & 2.75 & 2.76 & 2.78 & 2.79 & 2.81 & 2.83 \\
\hline $\mathrm{K}_{2} \mathrm{O}$ & 1.82 & 2.09 & 2.10 & 2.10 & 2.11 & 2.11 & 2.12 & 2.12 & 2.14 & 2.15 & 2.16 & 2.17 & 2.19 \\
\hline $\mathrm{P}_{2} \mathrm{O}_{5}$ & 0.4 & 0.46 & 0.46 & 0.46 & 0.46 & 0.46 & 0.47 & 0.47 & 0.47 & 0.47 & 0.47 & 0.48 & 0.48 \\
\hline $\mathrm{H}_{2} \mathrm{O}$ & & 2.75 & 2.70 & 2.64 & 2.59 & 2.54 & 2.48 & 2.43 & 2.32 & 2.21 & 2.10 & 1.99 & 1.88 \\
\hline $\mathrm{CaO} / \mathrm{Al}_{2} \mathrm{O}_{3}$ & & 0.50 & 0.49 & 0.49 & 0.49 & 0.49 & 0.49 & 0.49 & 0.48 & 0.48 & 0.47 & 0.47 & 0.47 \\
\hline Olivine vol.\% & & 0.01 & 0.00 & 0.00 & 0.00 & 0.00 & 0.00 & 0.00 & 0.00 & 0.00 & 0.00 & 0.00 & 0.00 \\
\hline Cpx vol.\% & & 11.80 & 11.90 & 12.00 & 12.20 & 12.30 & 12.50 & 12.60 & 13.00 & 13.30 & 13.60 & 14.00 & 14.40 \\
\hline Plag vol.\% & & 0.00 & 0.00 & 0.00 & 0.00 & 0.00 & 0.00 & 0.00 & 0.00 & 0.00 & 0.00 & 0.00 & 0.00 \\
\hline Vol.\% crystals & & 11.80 & 11.90 & 12.00 & 12.20 & 12.30 & 12.50 & 12.60 & 13.00 & 13.30 & 13.60 & 14.00 & 14.40 \\
\hline Fo\% & & 84.00 & & & & & & & & & & & \\
\hline
\end{tabular}

An\%

Bulk composition

\begin{tabular}{|c|c|c|c|c|c|c|c|c|c|c|c|c|c|}
\hline Input $\mathrm{H}_{2} \mathrm{O}$ & & 1.46 & 1.35 & 1.25 & 1.14 & 1.04 & 0.94 & 0.83 & 0.78 & 0.73 & 0.62 & 0.52 & 0.42 \\
\hline$P(\mathrm{MPa})$ & & 140 & 130 & 120 & 110 & 100 & 90 & 80 & 75 & 70 & 60 & 50 & 40 \\
\hline $\mathrm{SiO}_{2}$ & 50.9 & 51.11 & 51.21 & 51.32 & 51.43 & 51.57 & 51.78 & 51.99 & 52.10 & 52.20 & 52.43 & 52.65 & 52.88 \\
\hline $\mathrm{TiO}_{2}$ & 0.9 & 0.92 & 0.92 & 0.92 & 0.93 & 0.96 & 0.99 & 1.02 & 1.04 & 1.06 & 1.10 & 1.15 & 1.20 \\
\hline $\mathrm{Al}_{2} \mathrm{O}_{3}$ & 16.6 & 18.71 & 18.79 & 18.87 & 18.87 & 18.66 & 18.40 & 18.13 & 18.00 & 17.86 & 17.57 & 17.27 & 16.96 \\
\hline
\end{tabular}


Table 3 (continued)

\begin{tabular}{|c|c|c|c|c|c|c|c|c|c|c|c|c|c|}
\hline \multicolumn{14}{|c|}{ Bulk composition } \\
\hline $\mathrm{Fe}_{2} \mathrm{O}_{3}$ & & 2.47 & 2.47 & 2.48 & 2.49 & 2.55 & 2.61 & 2.67 & 2.70 & 2.74 & 2.81 & 2.88 & 2.96 \\
\hline $\mathrm{FeO}$ & 8.22 & 5.78 & 5.76 & 5.74 & 5.76 & 5.85 & 5.95 & 6.05 & 6.10 & 6.15 & 6.27 & 6.39 & 6.52 \\
\hline $\mathrm{MnO}$ & 0.16 & 0.19 & 0.20 & 0.20 & 0.20 & 0.20 & 0.21 & 0.21 & 0.21 & 0.22 & 0.22 & 0.22 & 0.23 \\
\hline $\mathrm{MgO}$ & 6.66 & 4.92 & 4.88 & 4.84 & 4.82 & 4.84 & 4.78 & 4.73 & 4.69 & 4.66 & 4.59 & 4.51 & 4.42 \\
\hline $\mathrm{CaO}$ & 10.9 & 8.61 & 8.55 & 8.49 & 8.40 & 8.28 & 8.19 & 8.09 & 8.04 & 7.98 & 7.87 & 87.75 & 7.62 \\
\hline $\mathrm{Na}_{2} \mathrm{O}$ & 2.39 & 2.84 & 2.86 & 2.88 & 2.91 & 2.93 & 2.96 & 2.99 & 3.00 & 3.01 & 3.03 & 3.04 & 3.04 \\
\hline $\mathrm{K}_{2} \mathrm{O}$ & 1.82 & 2.20 & 2.22 & 2.24 & 2.27 & 2.32 & 2.38 & 2.46 & 2.50 & 2.54 & 2.63 & 2.73 & 2.85 \\
\hline $\mathrm{P}_{2} \mathrm{O}_{5}$ & 0.4 & 0.48 & 0.49 & 0.49 & 0.50 & 0.51 & 0.53 & 0.54 & 0.55 & 0.56 & 0.59 & 0.61 & 0.64 \\
\hline $\mathrm{H}_{2} \mathrm{O}$ & & 1.76 & 1.65 & 1.53 & 1.42 & 1.33 & 1.23 & 1.13 & 1.08 & 1.03 & 0.91 & 0.80 & 0.67 \\
\hline $\mathrm{CaO} / \mathrm{Al}_{2} \mathrm{O}_{3}$ & & 0.46 & 0.46 & 0.45 & 0.45 & 0.44 & 0.44 & 0.45 & 0.45 & 0.45 & 0.45 & 5.08 & 0.45 \\
\hline Olivine vol.\% & & 0.00 & 0.00 & 0.00 & 0.00 & 0.10 & 0.46 & 0.82 & 1.01 & 1.19 & 1.56 & 1.95 & 2.33 \\
\hline Cpx vol.\% & & 14.80 & 15.30 & 15.70 & 16.10 & 16.30 & 16.10 & 16.00 & 16.00 & 16.00 & 16.00 & 16.10 & 16.30 \\
\hline Plag vol.\% & & 0.00 & 0.00 & 0.00 & 0.51 & 2.18 & 4.19 & 6.28 & 7.34 & 8.44 & 10.70 & 13.10 & 15.60 \\
\hline Vol.\% crystals & & 14.80 & 15.30 & 15.70 & 16.70 & 18.50 & 20.80 & 23.10 & 24.30 & 25.60 & 28.30 & 31.10 & 34.20 \\
\hline Fo\% & & & & & & 83.00 & 83.00 & 82.80 & 82.80 & 82.00 & 81.80 & 81.80 & 80.80 \\
\hline An\% & & & & & 81.80 & 80.80 & 79.80 & 78.80 & 78.80 & 77.80 & 76.80 & 75.50 & 73.70 \\
\hline \multicolumn{14}{|c|}{ Bulk composition } \\
\hline Input $\mathrm{H}_{2} \mathrm{O}$ & & & & 0.21 & & 0.10 & & 0.05 & & & & & 0.0010 \\
\hline$P(\mathrm{MPa})$ & & & & 20 & & 10 & & 5 & & & & & 0.1 \\
\hline $\mathrm{SiO}_{2}$ & & 50.9 & & 53.35 & & 53.58 & & 53.62 & & & & & 53.79 \\
\hline $\mathrm{TiO}_{2}$ & & 0.9 & & 1.35 & & 1.46 & & 1.61 & & & & & 1.63 \\
\hline $\mathrm{Al}_{2} \mathrm{O}_{3}$ & & 16.6 & & 16.30 & & 15.93 & & 16.11 & & & & & 15.55 \\
\hline $\mathrm{Fe}_{2} \mathrm{O}_{3}$ & & & & 3.14 & & 3.24 & & 3.16 & & & & & 3.34 \\
\hline $\mathrm{FeO}$ & & 8.22 & & 6.81 & & 6.98 & & 6.93 & & & & & 7.15 \\
\hline $\mathrm{MnO}$ & & 0.16 & & 0.24 & & 0.25 & & 0.29 & & & & & 0.26 \\
\hline $\mathrm{MgO}$ & & 6.66 & & 4.20 & & 4.06 & & 3.73 & & & & & 3.86 \\
\hline $\mathrm{CaO}$ & & 10.9 & & 7.33 & & 7.17 & & 6.81 & & & & & 7.00 \\
\hline $\mathrm{Na}_{2} \mathrm{O}$ & & 2.39 & & 3.00 & & 2.93 & & 2.91 & & & & & 2.80 \\
\hline $\mathrm{K}_{2} \mathrm{O}$ & & 1.82 & & 3.17 & & 3.40 & & 3.81 & & & & & 3.73 \\
\hline $\mathrm{P}_{2} \mathrm{O}_{5}$ & & 0.4 & & 0.73 & & 0.76 & & 0.92 & & & & & 0.90 \\
\hline $\mathrm{H}_{2} \mathrm{O}$ & & & & 0.38 & & 0.21 & & 0.12 & & & & & 0.00 \\
\hline $\mathrm{CaO} / \mathrm{Al}_{2} \mathrm{O}_{3}$ & & & & 0.45 & & 0.45 & & 0.42 & & & & & 0.45 \\
\hline Olivine vol.\% & & & & 3.15 & & 3.59 & & 3.00 & & & & & 4.03 \\
\hline Cpx vol.\% & & & & 17.60 & & 20.60 & & 23.60 & & & & & 24.20 \\
\hline Plag vol.\% & & & & 20.70 & & 21.70 & & 25.20 & & & & & 23.40 \\
\hline Vol.\% crystals & & & & 41.50 & & 45.90 & & 51.80 & & & & & 51.50 \\
\hline Fo\% & & & & 79.80 & & 78.80 & & 76.80 & & & & & 77.80 \\
\hline An\% & & & & 70.40 & & 68.00 & & 64.90 & & & & & 64.90 \\
\hline
\end{tabular}

\section{Constraining the Stromboli system}

Two distinct products are ejected at Stromboli; normal Strombolian explosions eject a crystal-rich, highly porphyritic (HP) black scoria, while the major eruptions and the most violent, paroxysmal, activity eject a low porphyritic (LP), volatile-rich, crystal-poor "golden" pumice in addition to the crystal-rich HP scoria (Francalanci et al., 1999). The bulk rock compositions of both the pumice and scoria are very similar, with 49-53 wt.\% $\mathrm{SiO}_{2}$ and 1.6-2.2 wt.\% $\mathrm{K}_{2} \mathrm{O}$ (Métrich et al., 2001; Landi et al., 2004), but have different crystal contents and glass compositions. The pumice contains $<10$ vol.\% (vesicle-free) microphenocrysts of olivine and clinopyroxene in a shoshonitic glass (Métrich et al., 2001, 2010), whereas the scoria have $45-55$ vol.\% (vesicle-free) crystals, made up of 10-17 vol.\% clinopyroxene, 2-6 vol.\% olivine and are dominated by $\sim 27-38$ vol.\% plagioclase (Métrich et al., 2001; Francalanci et al., 2004; Landi et al., 2004; Métrich et al., 2010). As a result of crystallisation, the glasses of the scoria are more evolved than those of the pumice, with $\mathrm{SiO}_{2}$ contents ranging from 51 to 53 wt.\% and $\mathrm{K}_{2} \mathrm{O}$ contents from 3.5 to 5.2 wt.\% (Francalanci et al., 2005).

Melt inclusions (MIs) trapped in olivine provide information on the chemical and volatile composition of the melt at the depth at which the MI formed. Olivine-hosted MIs found in the pumice at Stromboli have high volatile contents, 2.3-3.8 wt.\% $\mathrm{H}_{2} \mathrm{O}$ and 894-1704 ppm $\mathrm{CO}_{2}$ (Métrich et al., 2001, 2010), whereas the olivine-hosted MIs in the scoria are more extensively degassed, $0.4-1.1 \mathrm{wt} . \% \mathrm{H}_{2} \mathrm{O}$ and $273-473 \mathrm{ppm}$ $\mathrm{CO}_{2}$ (Métrich et al., 2010). This leads Métrich et al. $(2001,2010)$ to conclude that the MIs in the scoria are a result of decompression-driven degassing and crystallisation of the volatile-rich magma represented by the MI in the golden pumice.

\subsection{Modelling the degassing path}

Assuming equilibrium and volatile saturation, entrapment pressures for the MI compositions given by Métrich et al. (2010) are calculated using VolatileCalc (Newman and Lowenstern, 2002), which has been shown to describe the solubility of $\mathrm{CO}_{2}$ and $\mathrm{H}_{2} \mathrm{O}$ bearing fluids in Strombolian basalts well (Lesne et al., 2011; Witham et al., 2012). Entrapment pressures for $\mathrm{MI}$ in the scoria are found to range from 75 to $110 \mathrm{MPa}$ and in the pumice from 210 to $340 \mathrm{MPa}$ at $1130{ }^{\circ} \mathrm{C}$ (Fig. 2). Although simple open- or closed-system equilibrium degassing models (e.g. Dixon, 1997; Newman and Lowenstern, 2002; Papale et al., 2006) agree well with experimentally observed degassing paths, the models fail to reproduce the degassing trends represented by the MI; they underestimate the amount of dissolved $\mathrm{CO}_{2}$ in the melt. This could be explained by fluxing of $\mathrm{CO}_{2}$ through the magma from depth which would drive dehydration of the melt (Blundy et al., 2010; Métrich et al., 2010), or by mixing of ascending and descending magmas as discussed by Witham (2011a). Here we are interested only in the volatile content of the melt as a function of pressure and not the mechanism of the degassing path. Witham (2011b) highlights that if there is mixing between the two magmas, some melt may be undersaturated in volatiles. Here we follow Métrich et al. (2010) in assuming that the melt is volatile saturated and perform a simple empirical linear fit to the $\mathrm{X}_{\mathrm{H}_{2} \mathrm{O}}-P$ and $\mathrm{X}_{\mathrm{CO}_{2}}-P$ data where $\mathrm{X}$ is the concentration (wt.\%) and $P$ is the pressure (MPa), such that $\mathrm{X}_{\mathrm{H}_{2} \mathrm{O}}=0.0105 \cdot P$ and $\mathrm{X}_{\mathrm{CO}_{2}}=5.17 \cdot P$ (Fig. 2). This approach allows us to reproduce well the composition and crystallinity of the pumice and scoria ejected at Stromboli, as we discuss in Section 3.2.

We calculate the saturation pressure of the most primitive MI given by Métrich et al. (2010) (ST82 139a 3.5 wt.\% $\mathrm{H}_{2} \mathrm{O}, 1704$ ppm CO $\mathrm{CO}_{2}$ and 
47.5 wt.\% $\mathrm{SiO}_{2}$ ) using VolatileCalc (Newman and Lowenstern, 2002) to be $336 \mathrm{MPa}$ for which there is a $\mathrm{CO}_{2} / \mathrm{H}_{2} \mathrm{O}$ weight ratio in the equilibrium gas phase of 3.71. From Fourier Transform Infrared Spectroscopy (FTIR) measurements of gas $\mathrm{CO}_{2} / \mathrm{SO}_{2}$ molar ratios (M. Burton et al., 2007) during persistent degassing, M.R. Burton et al. (2007) estimate the total primitive $\mathrm{CO}_{2}$ content to be $2.44 \mathrm{wt}$.\%, assuming bulk magma degassing. Assuming closed-system degassing this suggests that at $336 \mathrm{MPa}$, 2.27 wt.\% $\mathrm{CO}_{2}$ and 0.716 wt.\% $\mathrm{H}_{2} \mathrm{O}$ are already in the gas phase. This would require a total primitive $\mathrm{H}_{2} \mathrm{O}$ content of $4.22 \mathrm{wt}$.\%. Given these total primitive contents of $\mathrm{H}_{2} \mathrm{O}$ and $\mathrm{CO}_{2}$ and the wt.\% of dissolved volatiles in the melt (Fig. 2) we can calculate the wt.\% of exsolved volatiles as a function of pressure. To determine the volume occupied by the exsolved gas we use the equation of state for $\mathrm{H}_{2} \mathrm{O}$ and $\mathrm{CO}_{2}$ given by Pitzer and Sterner (1994). We assume the magma will become permeable to gas flow at 50 vol.\% bubbles in the magma (melt + bubbles + crystals) following M.R. Burton et al. (2007), which is found to occur at $75 \mathrm{MPa}$, from which point the vesicularity of the magma is assumed to remain constant (equal to 50 vol.\%) and any extra gas is lost from the system.

\subsection{Modelling the crystallisation path}

The crystallisation paths and chemical compositions of the melts for both the ascending and descending magmas are modelled as a function of pressure using MELTS (Ghiorso and Sack, 1995; Asimow and Ghiorso, 1998). We use the whole rock composition of pumice ST82 (Métrich et al., 2010) and assume that the system is in equilibrium and isothermal, and that there is no fractional crystallisation. We fix $\mathrm{fO}_{2}$ at $\triangle \mathrm{NNO}+1$. MELTS does not account for $\mathrm{CO}_{2}$, which acts to lower the effective solubility of $\mathrm{H}_{2} \mathrm{O}$. We assume that $\mathrm{CO}_{2}$ does not affect crystallisation other than by reducing the activity of water in the melt, and thus is inert in regard to the crystal phase assemblage (e.g. Pichavant et al., 2009). Further we follow Dixon and Stolper (1995) in assuming ideal mixing in the fluid phase. To model the ascending magma we run MELTS in a step-wise manner, with the activity of $\mathrm{H}_{2} \mathrm{O}$ in the melt calculated using the linear fit of $\mathrm{X}_{\mathrm{H}_{2} \mathrm{O}}-P$ (Fig. 2) from 350 to $0.1 \mathrm{MPa}$ (over 53 pressure steps). As the fluid phase in MELTS is pure water only, the programme considers our modelled melt to be undersaturated in $\mathrm{H}_{2} \mathrm{O}$. This, however, allows us to represent well a melt saturated in a mixed $\mathrm{CO}_{2}$ and $\mathrm{H}_{2} \mathrm{O}$ fluid phase. We assume that the descending magma has no bubbles, a reasonable approximation for magma density and viscosity calculations given that any remaining bubbles would resorb back into the melt as the pressure increases. The $\mathrm{H}_{2} \mathrm{O}$ content of the descending magma is assumed to be constant and taken to be that of the ascending magma at the lowest pressure achieved before the magma overturns. Optical thermometry experiments on the MIs suggest homogenisation temperatures ranging from 1160 to $1110{ }^{\circ} \mathrm{C}$ (Métrich et al., 2001); we find that a temperature of $1130{ }^{\circ} \mathrm{C}$ results in crystallinities and melt compositions that represent well the properties of the pumice and scoria as a function of pressure (Figs. 3 and 4). Data from all of the MELTS runs and the bulk composition of pumice ST82 (Métrich et al., 2010) are given in Tables 1, 2, and 3.

Our modelled melt at $350 \mathrm{MPa}$ has a $\mathrm{SiO}_{2}$ content of $49 \mathrm{wt} . \%$ and $\mathrm{K}_{2} \mathrm{O}$ content of $2 \mathrm{wt} . \%$, and at $0.1 \mathrm{MPa}$ a $\mathrm{SiO}_{2}$ content of $53 \mathrm{wt} . \%$ and $\mathrm{K}_{2} \mathrm{O}$ content of $3 \mathrm{wt} . \%$. Actual olivines in the Stromboli pumice span a range of compositions from $\mathrm{FO}_{91-84}$ and those in the scoria range from $\mathrm{Fo}_{69-70}$ (Métrich et al., 2010). The olivine output from our petrologic modelling has a composition ranging from $\mathrm{Fo}_{86-85}$ over pressures of 350-270 MPa, which agrees well with the natural olivines. At pressures $\leq 90 \mathrm{MPa}$, the modelled olivine chemistry is slightly more forsteritic $\left(\mathrm{Fo}_{68-81}\right)$ than natural olivine in scoria $\left(\mathrm{Fo}_{69-70}\right)$. This is not significantly improved by using $\mathrm{T}=1115^{\circ} \mathrm{C}$ which results in compositions ranging from $\mathrm{Fo}_{65}-80$ over 100-0.1 MPa (for which olivine is stable at this temperature). Taking the density of olivine to be $3300 \mathrm{~kg} \mathrm{~m}^{-3}$, clinopyroxene to be $3400 \mathrm{~kg} \mathrm{~m}^{-3}$ and plagioclase to be $2800 \mathrm{~kg} \mathrm{~m}^{-3}$ (Ahrens, 1995) the crystal content in the melt, excluding bubbles, at $350 \mathrm{MPa}$ is 5 vol.\%

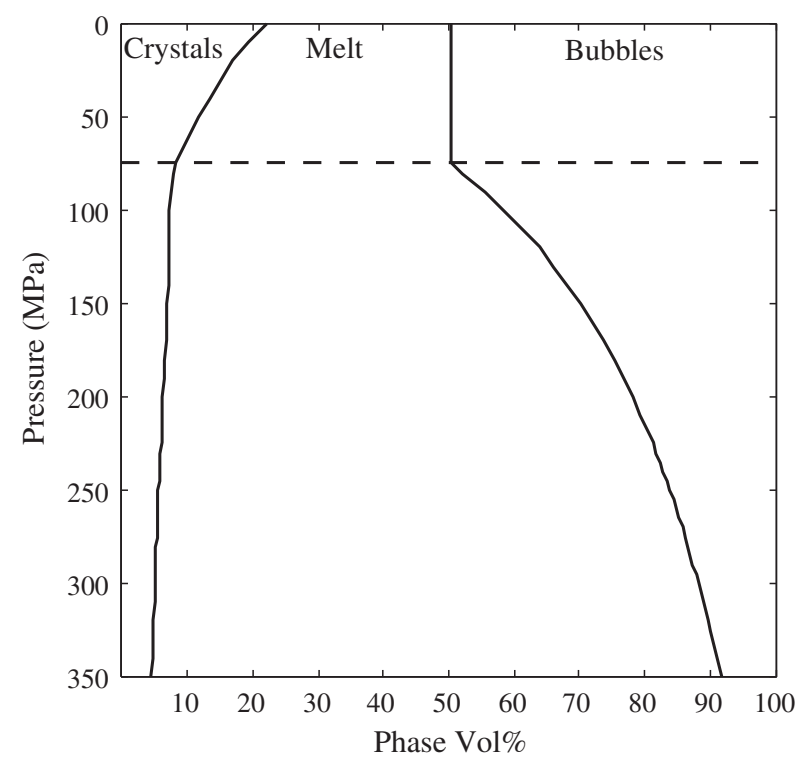

Fig. 5. Volume percent of crystals, melt and bubbles in the ascending magma at Stromboli. The dashed line indicates the onset of open-system degassing at $75 \mathrm{MPa}$, where the vesicularity of the magma has reached $50 \mathrm{vol} . \%$.

made up of olivine and clinopyroxene. In our model, plagioclase begins to form at $90 \mathrm{MPa}$ and by $40 \mathrm{MPa}$ the crystallinity is $27 \mathrm{vol} . \%$ ( $1 \mathrm{vol} . \% \mathrm{ol}-$ ivine, 14 vol.\% clinopyroxene, 12 vol.\% plagioclase). A final crystal content of 44 vol.\% (3 vol.\% olivine, 16 vol.\% clinopyroxene and 25 vol.\% plagioclase) was achieved at $0.1 \mathrm{MPa}$. As the $\mathrm{H}_{2} \mathrm{O}$-undersaturated magma returns back down the conduit it further crystallises upon descent, as the solidus temperature increases with an increase in pressure; at $350 \mathrm{MPa}$ magma which has descended from $0.1 \mathrm{MPa}$ has a crystallinity of 74 vol.\%. Comparing the volume percent of each crystal phase from the petrologic modelling to the crystal contents of scoria presented by Métrich et al. (2010) it is evident that there is a slight mismatch between the petrologic modelling and that observed in the scoria ejected at Stromboli: the vol.\% of plagioclase is slightly underestimated by the petrologic modelling with 24 vol.\% at 0.1 MPa compared to 2738 vol.\% found in the scoria (Fig. 4). This is not improved significantly by using a bulk composition of scoria (ST82s) (Métrich et al., 2001) which results in 27 vol.\% plagioclase at $0.1 \mathrm{MPa}$. Neither is it substantially improved by lowering the temperature from $1130{ }^{\circ} \mathrm{C}$ to $1115^{\circ} \mathrm{C}$, which results in a total crystallinity of 55 vol.\%, with only 23 vol.\% represented by plagioclase at $0.1 \mathrm{MPa}$.

In this study we calculate magma saturation pressures for the MI in the pumice to range from 210 to $340 \mathrm{MPa}$ at a temperature of $1130{ }^{\circ} \mathrm{C}$ using VolatileCalc (Newman and Lowenstern, 2002), which has recently been shown to best represent the solubility of $\mathrm{H}_{2} \mathrm{O}$ and $\mathrm{CO}_{2}$ bearing fluids in Stromboli basalt (Lesne et al., 2011; Witham et al., 2012). Using the VolatileCalc model (Newman and Lowenstern, 2002) results in greater saturation pressures for the MI in the pumice than those obtained using the (Papale et al., 2006) model: 130-250 MPa (Métrich et al., 2010). Using MELTS we find that $\mathrm{T}=1130{ }^{\circ} \mathrm{C}$ best represents crystal volume fractions, melt composition and mineral chemistry of the Stromboli magma over 350-0.1 MPa. This is a lower temperature than has been determined by crystallisation experiments conducted by Di Carlo et al. (2006) using a sample of pumice from Stromboli (PST 9, $\mathrm{SiO}_{2} 49.4$ wt.\% $\mathrm{K}_{2} 1.85$ wt.\%) who constrained pre-eruptive conditions to be $100-270 \mathrm{MPa}$ and $1140-1160{ }^{\circ} \mathrm{C}$ with $2.5-2.7 \mathrm{wt} . \% \mathrm{H}_{2} \mathrm{O}$, and also later Pichavant et al. (2009) who further constrained the preeruptive conditions of PST- 9 to be $1150{ }^{\circ} \mathrm{C}$ and $150-250 \mathrm{MPa}$. As a result we suggest deeper pre-eruptive storage conditions for LP magma of 8 $12 \mathrm{~km}$ (using an average crustal density of $2700 \mathrm{~kg} \mathrm{~m}^{-3}$ following Métrich et al. (2010)) compared to 7-10 km concluded by Métrich 
et al. (2010), and $\sim 8 \mathrm{~km}$ by Di Carlo et al. (2006) and Pichavant et al. (2009).

Métrich et al. (2010) also used MELTS to compute the crystallisation conditions of olivine; they found that olivine $\mathrm{Fo}_{89.8-88.7}$ joins the liquidus phase with clinopyroxene in equilibrium with a melt having $\sim 2.9$ wt.\% $\mathrm{H}_{2} \mathrm{O}$ and $~ 8.1$ wt.\% $\mathrm{MgO}$ between 300 and $250 \mathrm{MPa}$, and that the two phases start crystallising between 1190 and $1185{ }^{\circ} \mathrm{C}$ at $\triangle \mathrm{NNO}+1$, but they note that the temperature of crystallisation may be unreliable due to MELTS not treating the effect of $\mathrm{CO}_{2}$ on the phase equilibria. Here we represent a melt saturated in a mixed $\mathrm{CO}_{2}$ and $\mathrm{H}_{2} \mathrm{O}$ fluid phase by forcing MELTS to be undersaturated in $\mathrm{H}_{2} \mathrm{O}$. We find that olivine is only stable over $275-350 \mathrm{MPa}$ and $0-75 \mathrm{MPa}$ at $\mathrm{T}=$ $1130{ }^{\circ} \mathrm{C}$. Given that MIs are exclusively from olivine this explains the discontinuous nature of the MI saturation pressures.

We have calculated the volume percent of gas exsolved in the ascending magma (Section 3.1) and the volume percent of crystals in the melt. It is then important to define the volume percent of each phase as a function of the total volume of the melt, crystals and bubbles. In Fig. 5 the volume percent of crystals, bubbles and melt is re-calculated relative to the total volume of melt + bubbles + crystals. It is shown that at $0.1 \mathrm{MPa}$ the crystallinity of the ascending magma is now defined as 22 vol.\%, as melt occupies 28 vol.\% and gas occupies 50 vol.\% of the total volume.

\section{Physical properties of Strombolian magma}

\subsection{Magma rheology}

Calculating the viscosity of a three phase fluid (crystals, melt, bubbles) is challenging, and to date there is no complete model for this. We therefore exploit knowledge on the 2-phase end members, of a bubble suspension and of a crystal suspension. We explore two approaches: in the first we calculate the viscosity of the magma by first calculating the viscosity of the bubble-melt suspension, and use this as the effective suspending fluid in the rheological model for the crystal suspension; in the second approach we first calculate the viscosity of the crystal-melt suspension and use this as the effective suspending fluid in the rheological model for the bubble suspension. The limitation of this method is that we do not account for bubble-crystal interactions on the final viscosity of the system.

We use the model of Giordano et al. (2008) to calculate the viscosity of the silicate melt as a function of its chemical composition (determined from the MELTS calculations) at a temperature of $1130{ }^{\circ} \mathrm{C}$ and fluorine content of 0.1 wt.\% (Métrich et al., 2001) (Fig. 6).

For steady flow the rheology of the bubble-melt suspension depends on the melt viscosity, abundance of bubbles and the shape of the bubbles. Bubble shape depends on the Capillary number $(\mathrm{Ca})$, which defines the balance of viscous stresses which act to deform the bubbles and interfacial stresses which act to restore them to sphericity:

$C a=\lambda \gamma$

where $\lambda=\mu R_{b} / \Gamma$ is the bubble relaxation time, $\mu$ is the viscosity of the suspending fluid (the melt), $R_{b}$ is the radius of the bubble found to range from $10^{-2}$ to $10^{-5} \mathrm{~m}$ in the pumice and scoria ejected at Stromboli (Lautze and Houghton, 2007), $\Gamma$ is the liquid-bubble interfacial tension which is $\sim 0.25 \mathrm{~N} \mathrm{~m}^{-1}$ for basaltic magma (Murase and McBirney, 1973), and $\dot{\gamma}$ is strain rate.

We can estimate a characteristic strain rate for an exchange flow at Stromboli, given the volume flux $(Q)$ of the flow:

$\dot{\gamma}=Q / r_{L}^{3}$,

where $r_{L}$ is the characteristic length scale and depends on the flow geometry. For SBS flow we take $r_{L}=r_{2}$, where $r_{2}$ is the conduit radius. For CAF $r_{L}=r_{1}$ for the inner (core) fluid, where $r_{1}$ is the radius of the

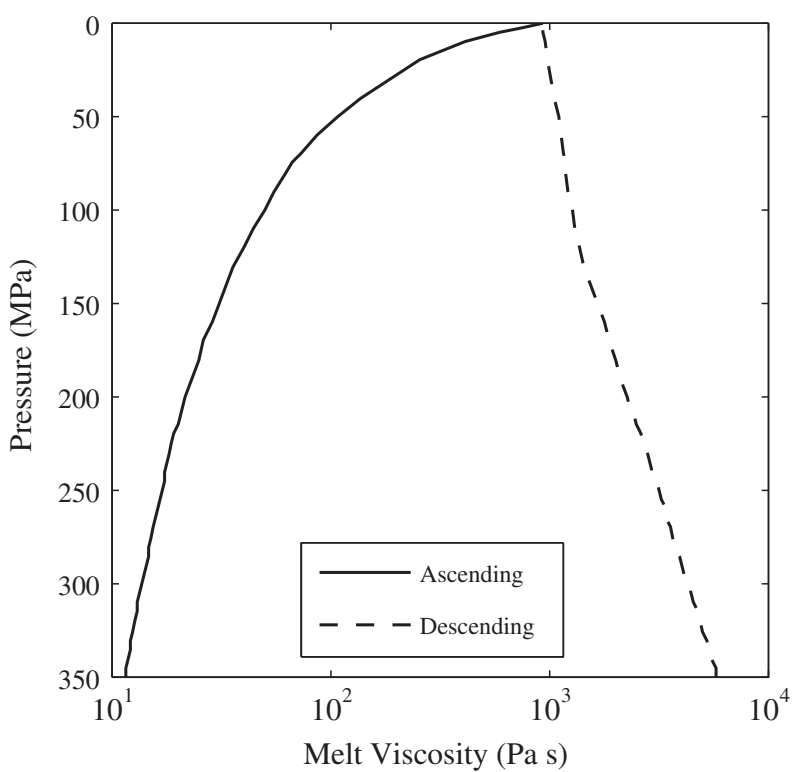

Fig. 6. Calculated viscosities of ascending and descending melt at Stromboli.

core, and for the descending outer fluid $r_{L}=r_{2}$. We assume that the flow is steady and that $Q$ and thus $\dot{\gamma}$, do not change substantially with depth in the conduit; in Section 5.1 we show that the decrease in magma density during ascent reduces $Q$ by less than an order of magnitude.

Eq. (3) does not account for variation in strain rate with radial position in the conduit. To validate this approach we apply Eq. (3) to laboratory exchange flows presented by Beckett et al. (2011) and compare to the actual strain rates calculated from flow velocities, $\dot{\gamma}(r)=\frac{d u}{d r}$ (Figs. 7 and 8). For CAF $\dot{\gamma}(r)$ is calculated using the best fit velocity profiles to the theoretical solution of Huppert and Hallworth (2007) (Fig. 9; Beckett et al., 2011). For SBS flow $\dot{\gamma}(r)$ is calculated from high order polynomial fits to the measured data points because the available theoretical velocity profiles of Kerswell (2011) do not well represent the SBS experimental data (Fig. 10; Beckett et al., 2011).

Figs. 7 and 8 show that using the volume flux of the flow (Eq. (3)) gives useful order of magnitude estimates for strain rate. Given that the minimum observed radius of the ascending fluid in the CAF regime from the exchange flow experiments is $0.2 r_{2}$ (Table 2; Beckett et al., 2011) and taking the volume flux of the magma at Stromboli required to account for measured persistent gas fluxes to be $\sim 10^{-1} \mathrm{~m}^{3} \mathrm{~s}^{-1}$ we can estimate the strain rate acting on an exchange flow of magma in a conduit with radius $1-10 \mathrm{~m}$ to range from $10^{1}$ to $10^{-4} \mathrm{~s}^{-1}$.

$\mathrm{Ca}$ is now calculated for an exchange flow of magma at Stromboli as a function of pressure for a conduit with radius $1-10 \mathrm{~m}$ (i.e. $\dot{\gamma}=$ $10^{1}-10^{-4} \mathrm{~s}^{-1}$ ) (Fig. 9). For most of the conditions considered $\mathrm{Ca} \ll 1$, except for the extreme case where $r_{2}=1 \mathrm{~m}$ and $R_{b}=10^{-2} \mathrm{~m}$ for which $C a \leq O$ (100). However, taking $C a<1$ would predict relative viscosities for $\mathrm{Ca} \leq 100$ within an order of magnitude for $\phi_{b} \leq 0.5$, where $\phi_{b}$ is the bubble volume fraction (Pal, 2004). We therefore assume that $C a \leq O$ (1) best represents the flow: the bubbles remain spherical and with an increase in bubble volume fraction $\left(\phi_{b}\right)$ viscosity increases Rust and Manga, 2002. The rheology of bubble suspensions for low $\mathrm{Ca}$ number is given by (Pal, 2002; Llewellin and Manga, 2005; Mader et al., 2013):

$\mu_{r}=\left(1-\phi_{b}\right)^{-1}$

where $\mu_{r}$ is relative viscosity, normalised to that of the interstitial fluid, and $\phi_{b}$ is the volume fraction of bubbles. 
a)

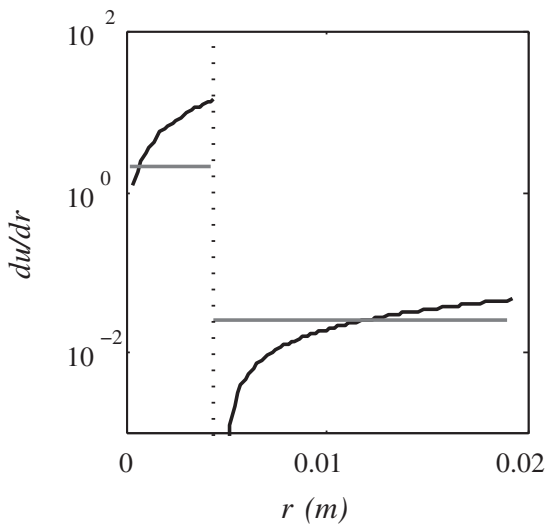

c)

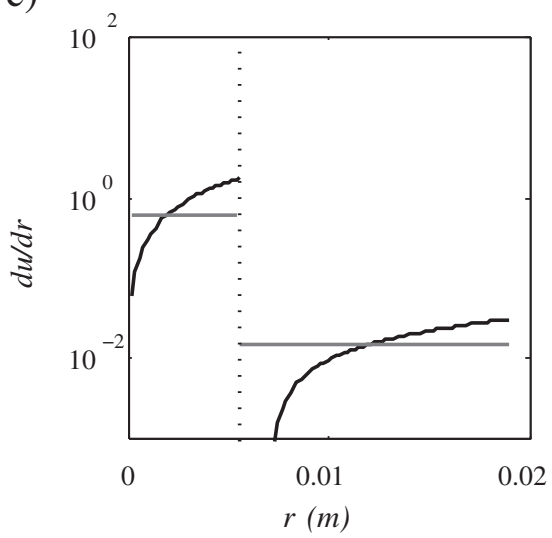

b)

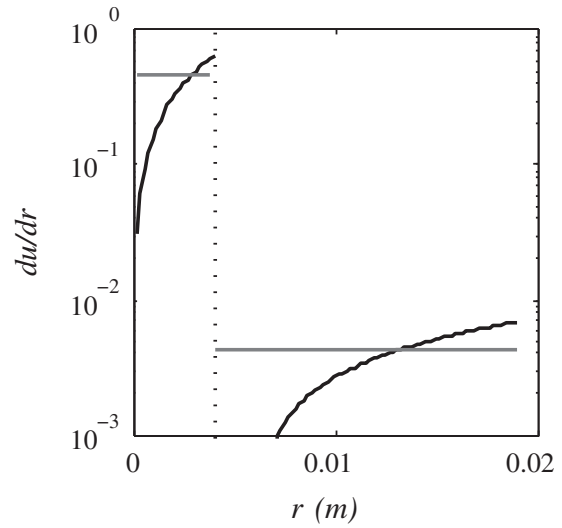

d)

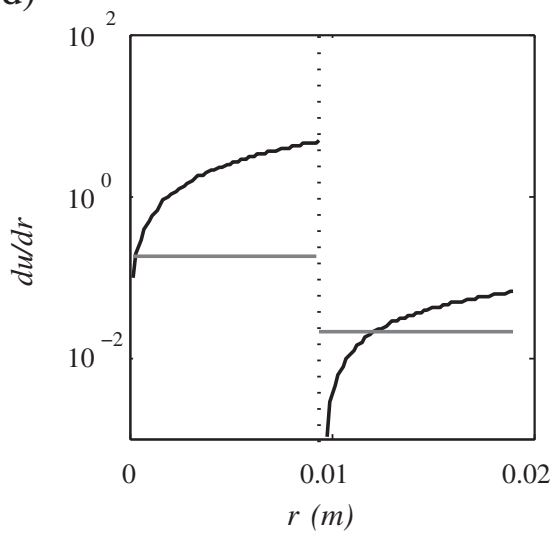

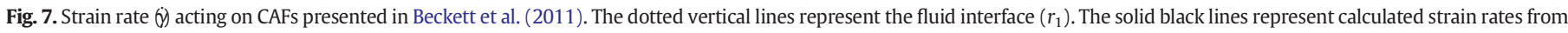

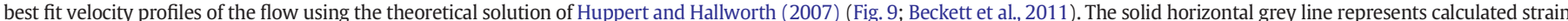

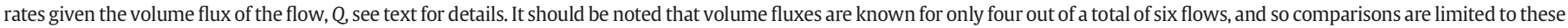
experiments.

There are a number of published models to describe the rheological behaviour of fluids with a crystal volume fraction $(\phi)$ (e.g. Costa, 2005; Caricchi et al., 2007; Costa et al., 2009; Mueller et al., 2010). At low crystal contents the parameterisations of Mueller et al. (2010) and Costa et al. (2009) are very similar, however as the Costa et al. (2009) model extends to higher crystal fractions we shall use their semi-

a)

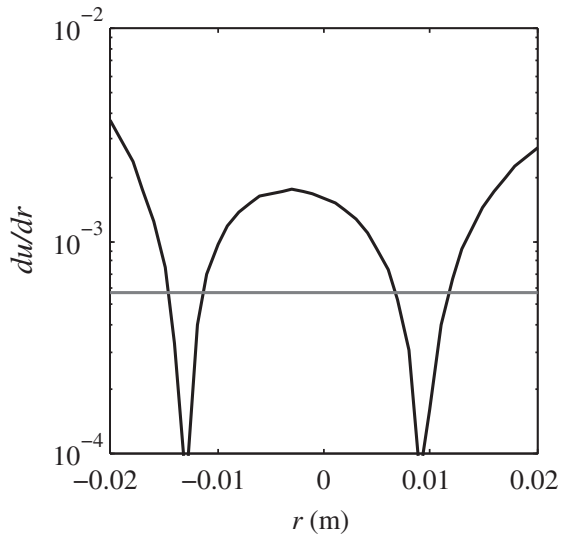

empirical parameterisation to calculate the relative viscosity of the crystal suspension:

$\eta_{r}=\frac{1+\varphi^{\delta}}{[1-F(\varphi, \varepsilon, \zeta)]^{5 / 2 \phi^{*}}}$

b)

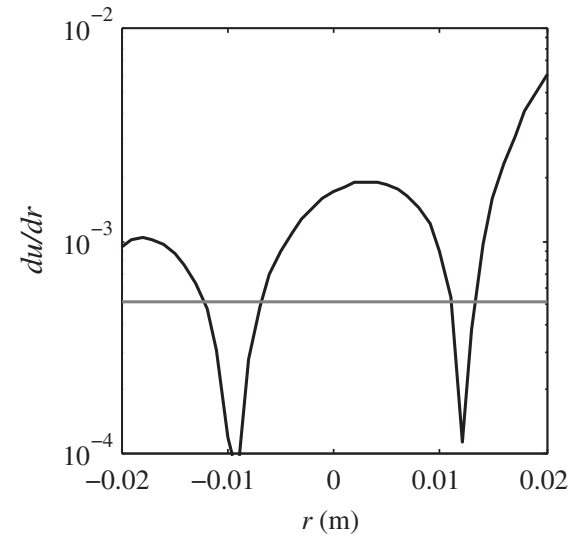

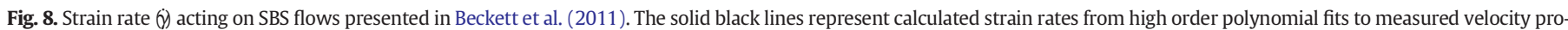
files (Fig. 10; Beckett et al., 2011). The solid horizontal grey line represents calculated strain rates from the volume flux of the flow, $Q$, see text for details. 


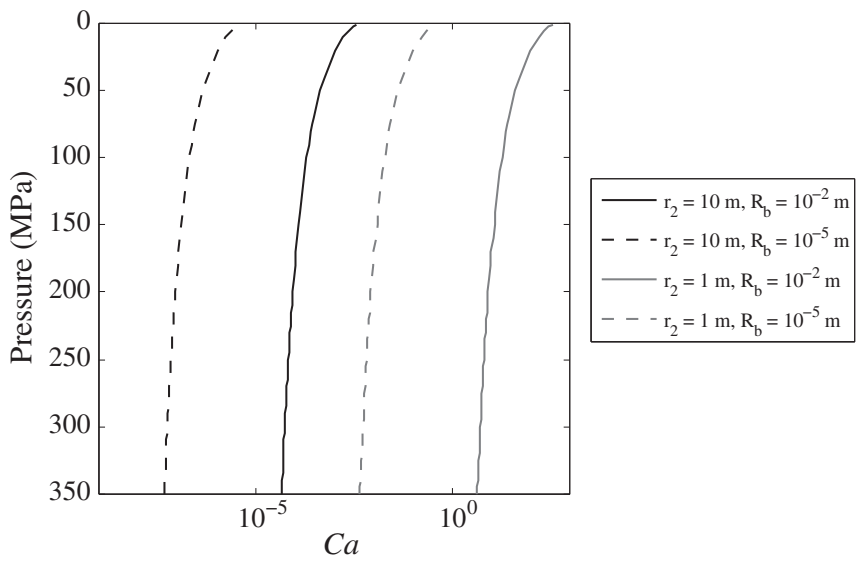

Fig. 9. Capillary number $(\mathrm{Ca})$ of the bubble suspension in ascending melt at Stromboli as a function of pressure, for conduit radii of $r_{2}=1 \mathrm{~m}$ and $r_{2}=10 \mathrm{~m}$ and bubble radii ranging from $R_{b}=10^{-5}$ to $10^{-2} \mathrm{~m}$.

$F=(1-\xi) \cdot \operatorname{erf}\left[\frac{\sqrt{\pi}}{2 \cdot(1-\xi)} \varphi \cdot\left(1+\varphi^{\zeta}\right)\right]$

For a critical solid fraction $\left(\phi^{*}\right)$ the viscosity of the suspension tends to a constant value, where $\varphi=\phi / \phi^{*}$ and $\xi, \zeta, \delta$ and $\phi^{*}$ are empirical parameters given by Caricchi et al. (2007) as a function of strain rate $\dot{\gamma}$ for suspensions of isotropic particles with $\phi$ ranging from 0.1 to 0.8 .

The viscosities of the ascending and descending magma at Stromboli are shown in Fig. 10. There is no significant difference in the calculated viscosities of the ascending magma when using the crystals plus melt as the suspending fluid for bubbles compared to using the bubbles plus melt as the suspending fluid for crystals. At $350 \mathrm{MPa}$ the viscosity of the ascending magma is $\mu_{1} \sim 10^{1} \mathrm{~Pa}$ s and at $0.1 \mathrm{MPa} \mu_{1} \sim 10^{3} \mathrm{~Pa} \mathrm{~s}$, for a conduit with radius $1-10 \mathrm{~m}$. The viscosity of the descending magma at a given pressure depends on the pressure at which the magma is assumed to change from ascending to descending (Fig. 3) as this affects the water content of the descending magma which in turn affects its crystallinity. For example, if the magma has descended from $0.1 \mathrm{MPa}$, then at $350 \mathrm{MPa} \mu_{2} \sim 10^{9}-10^{10} \mathrm{~Pa} \mathrm{~s}$, but if it has descended from $75 \mathrm{MPa}$ (the point at which the vesicularity remains at 50 vol.\%) it has a viscosity of $\mu_{2} \sim 10^{2}$ Pa s.

\section{a) Ascending}

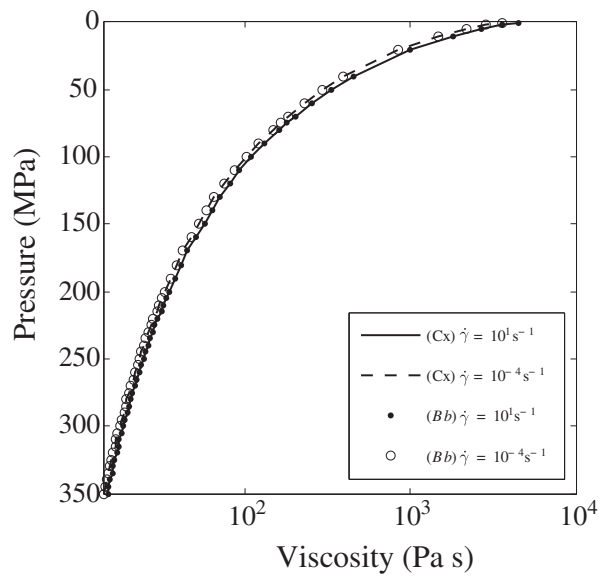

\subsection{Magma density}

The process of magma convection in the conduit is driven by the density difference between degassed crystallised magma at shallow levels and buoyant gas-rich magma deeper within the system. The densities of the ascending and descending magmas as a function of pressure can be calculated given the proportion of crystals, melt and bubbles (Fig. 11a). The density of the melt is taken to be $2700 \mathrm{~kg} \mathrm{~m}^{-3}$ (Richet et al., 2000), and the density of olivine is taken to be $3300 \mathrm{~kg} \mathrm{~m}^{-3}$, clinopyroxene $3400 \mathrm{~kg} \mathrm{~m}^{-3}$ and plagioclase $2800 \mathrm{~kg} \mathrm{~m}^{-3}$ (Ahrens, 1995). The vesicularity of the magma was calculated in Section 3.1. As magma ascends through the conduit volatile exsolution drives an increase in vesicularity which decreases the density of the magma. At $75 \mathrm{MPa}$ the vesicularity of the magma reaches $50 \mathrm{vol} \%$, and the magma is assumed to become permeable to gas flow (M.R. Burton et al., 2007). The magma continues to ascend (as it is less dense than the degassed magma) but its vesicularity now remains constant and any extra gas is lost from the system; its density now decreases only slightly due to changes in the density of the gas phase as a function of pressure. As magma descends back down the conduit after losing all of its gas, it continues to crystallise and its density increases with pressure. The calculated density difference between the ascending and descending magmas as a function of pressure is shown in Fig. 11b. Note that this local density contrast is different to the total vertical density difference across the length of the conduit, between the degassed magma at $0.1 \mathrm{MPa}$ and the buoyant ascending magma at $350 \mathrm{MPa}$, which is $\sim 1500 \mathrm{~kg} \mathrm{~m}^{-3}$.

\section{Exchange flow}

\subsection{Volume flux and conduit radius}

Following the method of M.R. Burton et al. (2007) the mass flux of magma required to account for the observed persistent gas flux at Stromboli of $200 \mathrm{t} \mathrm{d}^{-1} \mathrm{SO}_{2}$ is $565 \mathrm{~kg} \mathrm{~s}^{-1}$, taking the density of the melt to be $2700 \mathrm{~kg} \mathrm{~m}^{-3}$. Using our calculated densities for the magma as it ascends through the conduit we can calculate the volume flux of magma required to achieve a mass flow rate of $565 \mathrm{~kg} \mathrm{~s}^{-1}$ (Fig. 12). As gas exsolves the density of the magma decreases and the volume flux of ascending magma increases with a decrease in pressure. Using Eq. (1) we can calculate the minimum radius of the conduit required to account for this volume flux, and hence the observed gas flux, as a function of depth in the conduit. Using our modelled fluid properties at $0.1 \mathrm{MPa}$ suggests a conduit radius of $1.6 \mathrm{~m}$ (Fig. 13). This is similar b) Descending

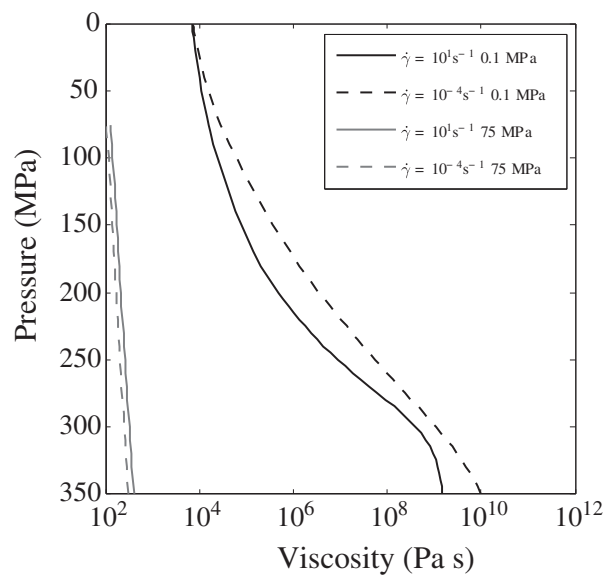

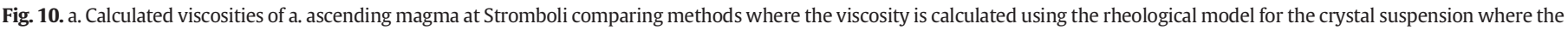

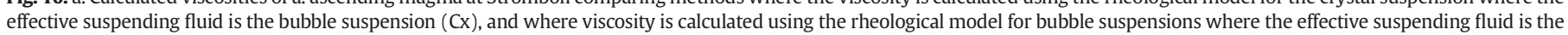
crystal suspension $(\mathrm{Bb})$. b. Calculated viscosities of descending magma (crystals-melt) at Stromboli from 0.1 MPa and 75 MPa at Stromboli. 
a)

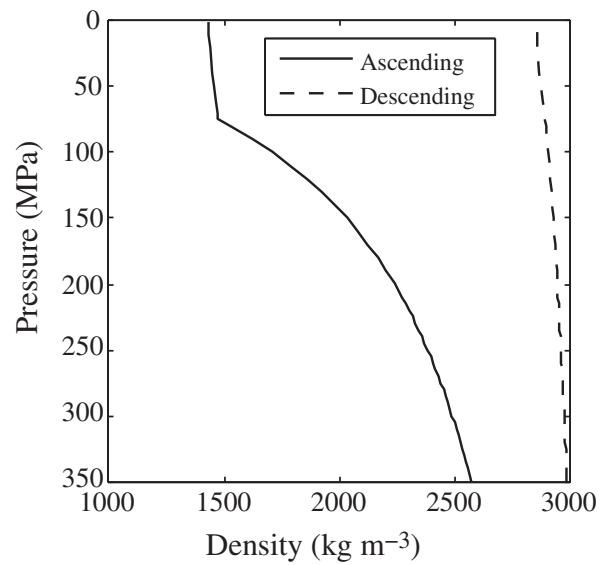

b)

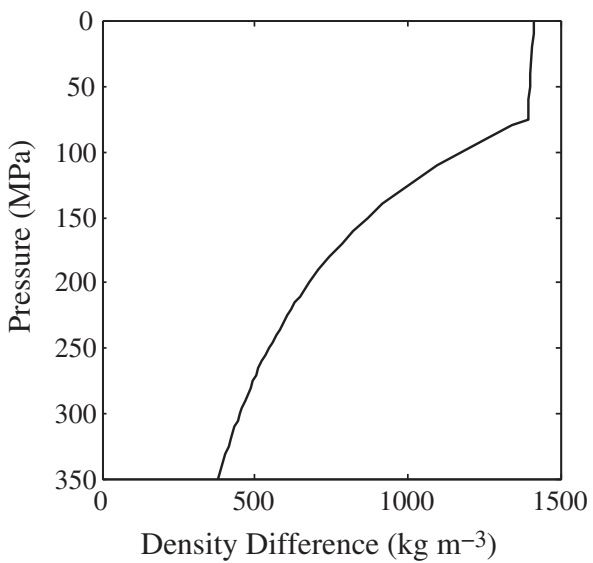

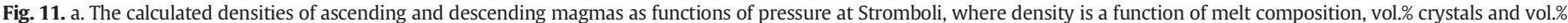
bubbles. $\mathrm{b}$. The density difference between ascending and descending magma as a function of pressure.

to that obtained by M.R. Burton et al. (2007) who employ the assumption of maximum flux, $r_{2}=1.25-1.45 \mathrm{~m}$, but for a larger mass flux of $575 \mathrm{~kg} \mathrm{~s}^{-1}$, and to measured vent diameters at Stromboli of 4-8 m (Harris and Stevenson, 1997; Ripepe et al., 2007). The minimum required conduit radius increases with increasing pressure due to systematic changes in magma viscosities and densities; with the magma properties at $350 \mathrm{MPa}$, the calculated conduit radius is $12-17 \mathrm{~m}$, although we do not expect the plumbing system to be a simple cylinder so deep in the system.

\subsection{Flow regime}

Assuming that the volume fluxes of the ascending and descending magma are matched, the Reynolds number of the more viscous fluid can be defined as:

$R e_{2}=\frac{Q \rho_{2}}{\mu_{2} r_{2}}$

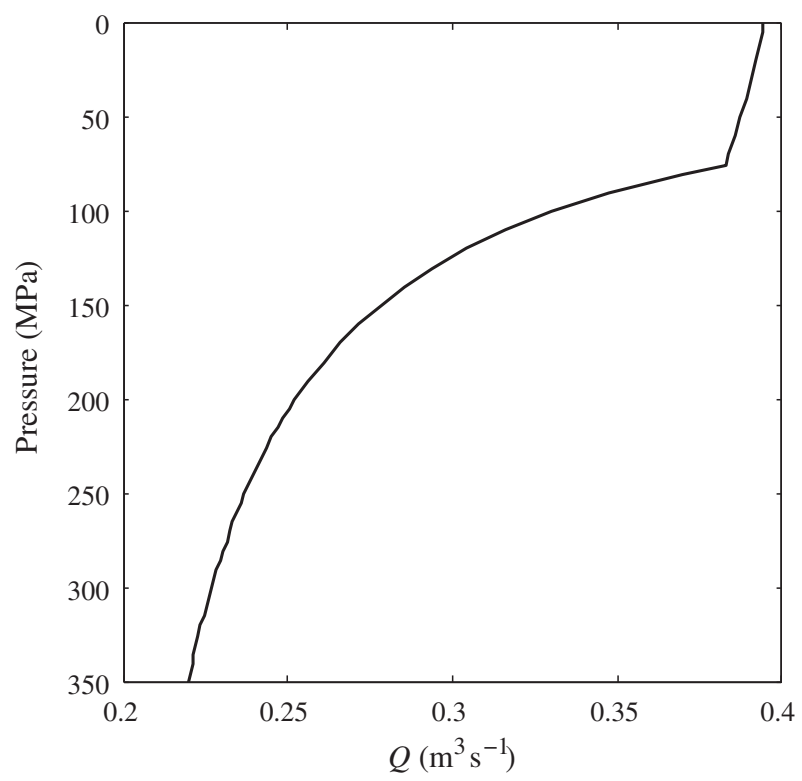

Fig. 12. The volume flux of ascending magma at Stromboli given that the mass flux of magma required to drive the persistent gas flux of $\sim 200 \mathrm{t} \mathrm{d}^{-1}$ of $\mathrm{SO}_{2}$ (Allard et al., 2008) is $565 \mathrm{~kg} \mathrm{~s}^{-1}$. The increase in volume flux, as magma ascends, is due to the decrease in density driven by gas exsolution and decompression. where $\rho_{2}$ and $\mu_{2}$ are the density and viscosity of the more viscous fluid. In Fig. 14 calculated $R e_{2}$ of the experimental exchange flows presented by Beckett et al. (2011) and those in the studies of Huppert and Hallworth (2007) and Palma et al. (2011) are compared to $R e_{2}$ calculated for Stromboli based on the descending magma properties calculated in this study and the required volume flux of magma needed to account for the measured persistent gas fluxes. For an exchange flow at Stromboli both $\mu_{2}$ and $\rho_{2}$ vary with pressure; consequently as pressure increases, $R e_{2}$ decreases and $\beta$ increases. Over 0.1-350 MPa, for a conduit with radius $1-10 \mathrm{~m}, R_{2}<0.3$, suggesting that the flow will be laminar and can be well described by the low Reynolds number exchange flows presented by Beckett et al. (2011).

In the conduit of a volcano the growth of bubbles and crystals causes the viscosities and buoyancies of the magmas to change with pressure. In the analogue exchange flow experiments viscosities and buoyancy forces are independent of depth in the pipe. To apply the experimental results to model the flow regime of magma driving persistent degassing at Stromboli we calculate the viscosity ratio between the ascending and

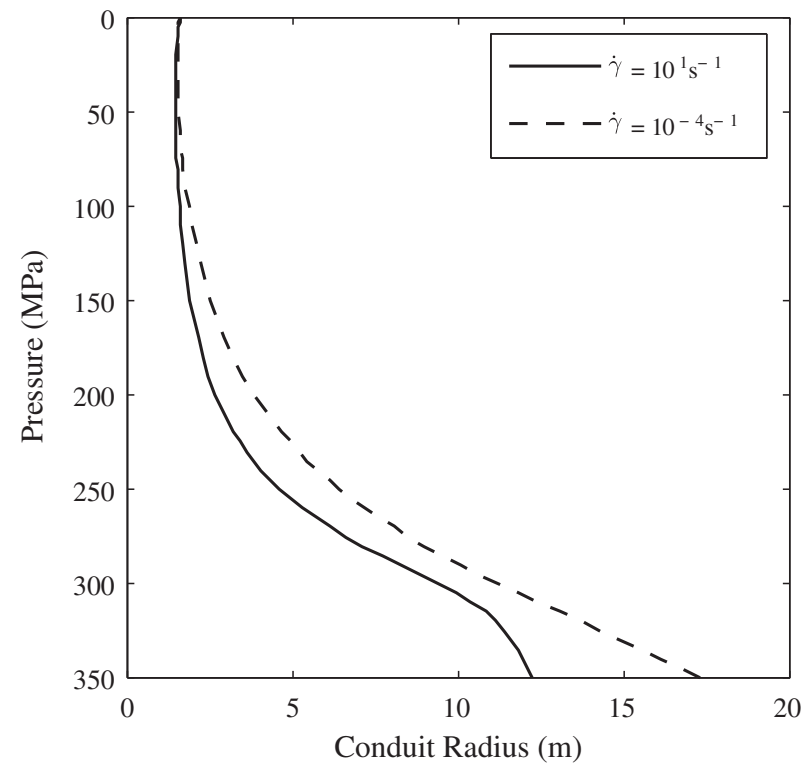

Fig. 13. The minimum conduit radius required to account for the volume flux of ascending magma at Stromboli needed to drive the persistent gas flux of $\sim 200 \mathrm{t} \mathrm{d}^{-1}$ of $\mathrm{SO}_{2}$ (Allard et al., 2008). The radius as a function of depth is determined from Eq. (1) using calculated magma properties, where the viscosity of ascending magma has been determined for strain rates $\dot{\gamma}=10^{1}-10^{-4} \mathrm{~s}^{-1}$. 


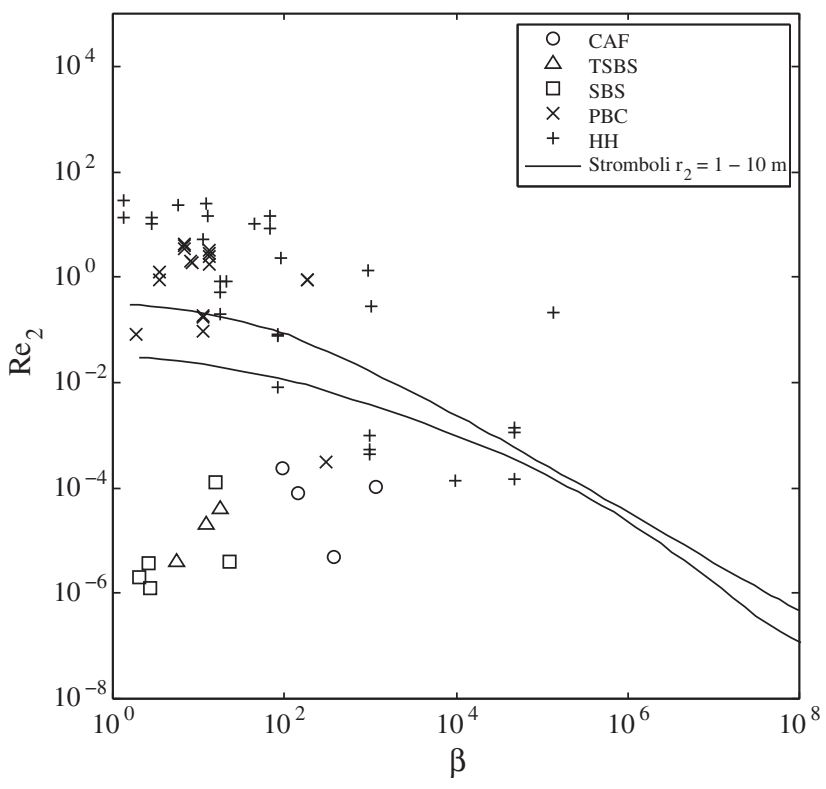

Fig. 14. Flow regimes as a function of $\beta$ and $R e_{2}$ of exchange flows observed by Beckett et al. (2011) $\left(r_{2}=19.2 \mathrm{~mm}\right)$ compared to the experimental studies by Huppert and Hallworth (2007) (HH) $\left(r_{2}=4,5.5,8,10,12\right.$ and $\left.15 \mathrm{~mm}\right)$ and Palma et al. (2011) (PBC) ( $r_{2}=10$ and $25 \mathrm{~mm}$ ), where $\operatorname{Re}_{2}$ is calculated using the known volume fluxes of the flow, Eq. (7). The solid lines represent exchange flow at Stromboli over 0.1-350 MPa, where $r_{2}=1-10 \mathrm{~m}$. As pressure increases in the conduit $R e_{2}$ decreases and $\beta$ increases. All $\operatorname{Re}_{2} \leq 0.3$, exchange flow at Stromboli is laminar.

descending magma as a function of pressure in the conduit, using the results from the rheology calculations in Section 4. Given that calculated viscosities using the crystal suspension as the effective suspending fluid in the rheological model for the bubble suspension did not differ significantly from calculated viscosities using the bubble suspension as the effective suspending fluid in the rheological model from the crystal suspension, we just use the latter method to calculate viscosity ratios. Assuming that the magma descends from $0.1 \mathrm{MPa}$, at $P \gtrsim 90 \mathrm{MPa}$, $\beta>100$ and the flow regime will be CAF, at $P \lesssim 90 \mathrm{MPa}, \beta<100$ and the flow regime will be SBS (Fig. 15a). In the experimental exchange flows Beckett et al. (2011) observed both regimes coexisting in the pipe separated by an abrupt transition (TSBS flow), with no step change in volume flux (compared to SBS flow at the same $\beta$ ), for $\beta=5-59$, suggesting that it is reasonable to expect that a flow regime transition could occur from CAF to SBS flow within the conduit. If the magma instead descends from $75 \mathrm{MPa}$ (the point at which the vesicularity of the magma remains at 50 vol.\%), $\beta<30$, due to the higher volatile content in the descending melt, and SBS will dominate below the level of overturn(Fig. 15b).

\section{Discussion and conclusions}

By constraining degassing and crystallisation paths at Stromboli using petrological and gas flux data we model the three-phase composition (crystals, melt, bubbles) of the ascending and descending magmas at Stromboli as a function of pressure. We calculate the rheology of this three-phase magma, accounting for its vesicularity, crystallinity and the composition of its melt, by employing recent studies on the rheology of crystal and bubble suspensions. We find that the viscosity of the ascending magma increases from $\sim 10^{1}$ to $\sim 10^{3}$ Pa $s$ from $350 \mathrm{MPa}$ to $0.1 \mathrm{MPa}$. The three-phase model of the viscosity of ascending and descending magma in conjunction with analogue exchange flow experiments results in a change in perspective of the flow regime of magma driving persistent degassing at Stromboli. We find that the viscosity ratio between the ascending and descending magma at Stromboli at $P \gtrsim 90 \mathrm{MPa}(\sim 3 \mathrm{~km})$ is $\gtrsim 100$ and so we predict the flow regime to be CAF, but at $P \lesssim 90 \mathrm{MPa}(\sim 3 \mathrm{~km})$ the viscosity ratio is $\lesssim 100$ and so the predicted flow regime is SBS. Although analogue exchange flow experiments show that the volume flux is independent of flow regime, the difference in melt velocity and viscosity distribution in the conduit between SBS and CAF could have important implications for the ascent of gas slugs driving explosive activity.

Regardless of the geometry of the exchange flow and conduit, the viscosity of the magma is highly dependent on its crystal volume fraction. Using a temperature of $1130{ }^{\circ} \mathrm{C}$ allows us to model the system well, resulting in crystallinities in accordance with those observed in the golden pumice and black scoria ejected at Stromboli, as discussed in Section 3.2. If we were to model the system with a lower temperature of $1115^{\circ} \mathrm{C}$, which is within the temperature range suggested from optical thermometry (Métrich et al., 2001), the crystallinity of the model system would increase. At $40 \mathrm{MPa}$ we would predict that 34 vol.\% crystals and the transition from CAF to SBS flow would occur at a pressure of $\sim 150 \mathrm{MPa}$.

We have assumed that the magma ascends to $0.1 \mathrm{MPa}$, before overturning and descending. If magma were to descend from $75 \mathrm{MPa}$ (the point at which the vesicularity of the magma remains at 50 vol.\%) it would have a lower crystallinity due to a higher water content in the melt, reducing the viscosity ratio between the ascending and descending magma such that SBS flow would dominate below the level of overturn. Further only $\sim 1 / 3$ of $S$ would degas (Lesne et al., 2011), thus to supply the observed $\mathrm{SO}_{2}$ flux the

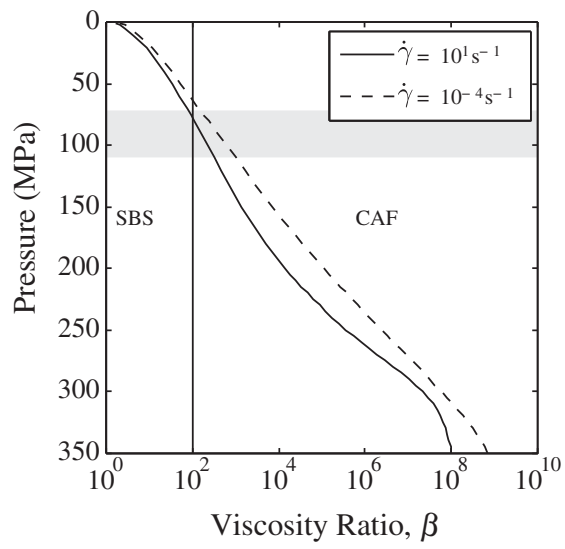

b)

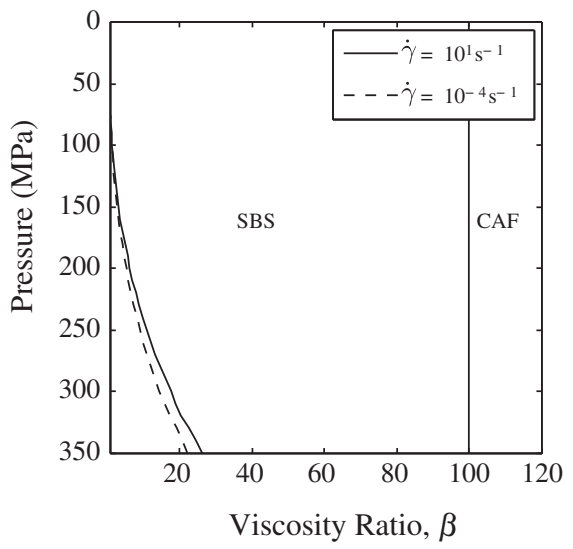

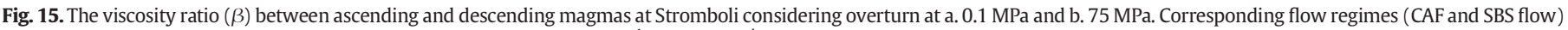

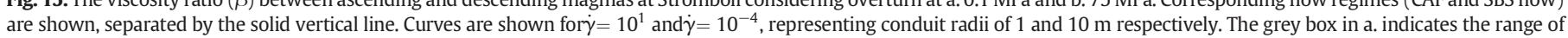
pressures where a change in regime is predicted for the suite of curves shown. 
magma flux would have to increase by a factor of 3 , and the radius of the conduit would increase to 2-23 m (0.1-350 MPa).

We assume that the gas phase is entirely lost before the magma descends, and thus no bubbles are transported in the descending magma and the magma becomes increasingly undersaturated as it descends. Diffusion of volatiles from volatile-rich ascending magma into the degassed descending magma would reduce the crystallinity of the descending magma, as the presence of volatiles reduces the liquidus temperature. To consider whether this process is significant we consider a characteristic length scale for diffusion given by $\sqrt{D t}$, where $D$ is diffusivity $\left(\mathrm{m}^{2} \mathrm{~s}^{-1}\right)$ and $t$ is time ( $\left.\mathrm{s}\right)$. Typical diffusivities for $\mathrm{H}_{2} \mathrm{O}$ in a basaltic melt are on the order of $10^{-9}-10^{-11} \mathrm{~m}^{2} \mathrm{~s}^{-1}$ for $1-3 \mathrm{wt}$ \% $\mathrm{H}_{2} \mathrm{O}$ at 0 $100 \mathrm{MPa}$ and $1300{ }^{\circ} \mathrm{C}$ (Persikov et al., 2010). We estimate the time scale for diffusion by considering how long it takes the magma to travel $1 \mathrm{~km}$ in the conduit. The velocity of the magma is $\sim 10^{-1} \mathrm{~m} \mathrm{~s}^{-1}$, based upon our calculated $Q \sim 10^{-1} \mathrm{~m}^{3} \mathrm{~s}^{-1}$ for Stromboli (Fig. 12), and that the radius of the ascending fluid is $\geq 0.2 r_{2}$ (Beckett et al., 2011). Thus for $1 \mathrm{~km}$ of flow in the conduit we have $t=10^{3} \mathrm{~s}\left(1000 \mathrm{~m} / 0.1 \mathrm{~m} \mathrm{~s}^{-1}\right)$ for diffusion, and so the length scale over which diffusion would occur is of the order $10^{-3}-10^{-4} \mathrm{~m}$. To allow homogenisation of volatiles despite such a short diffusion length scale would therefore require physical mixing between the volatile-rich ascending magma and the volatile poor descending magma. Using our calculated magma properties we showed that all $R e_{2}<0.3$, indicating that exchange flow at Stromboli is laminar (Fig. 14). We conclude that there will be very little physical mixing, and the length scales over which diffusion will occur in an exchange flow will be insignificant. However, a further observation from the analogue experiments presented by Beckett et al. (2011) is that a proportion of the less viscous fluid is dragged downwards with the more viscous fluid at the fluid interface. This could account for recycling of magma within the conduit as evidenced by mineral dissolution and reverse and/or oscillatory zoning of crystals in the pumice and scoria emitted at Stromboli (e.g. Landi et al., 2004; Francalanci et al., 2005; Landi et al., 2008).

The empirical relationship given by Beckett et al. (2011) is used in this study to constrain the radius of the conduit at Stromboli (Eq. (1)) from the magma volume flux based on $\mathrm{SO}_{2}$ emissions and calculations of the three-phase magma viscosities and densities. However, it is unlikely that the volume flux and magma properties, which will vary temporally, solely control the conduit radius. Instead this represents only the minimum radius required to account for the gas fluxes observed. M. Burton et al. (2007) note that the 'quiescent gas release between the explosions has a well-defined, quite steady mean composition'. However, if the bubble and/or crystal fraction in the ascending magma and hence the viscosity ratio between the ascending and descending magmas varies in time, then the depth of transition from SBS to CAF will also vary.

In the analogue experiments the volume flux of the ascending fluid is constrained to be equal to the net downwards flux of the more dense, more viscous fluid. Thus Eq. (1) also implicitly assumes that the volume fluxes $(Q)$ of the ascending and descending magmas are matched. Given that the vesicular ascending magma is less dense than the degassed descending magma, matching the volume flux means that the mass flux of the ascending magma is less than that of the descending magma. This is partly, but not entirely, offset by the several weight percent of volatiles that are released at shallow levels and do not descend with the degassed magma. Therefore matching of volume flux would drive the free surface level of the magma in the conduit to fall over time. Although a drop in the magma level is reasonable over short time scales (e.g. Ripepe et al., 2002; Lautze and Houghton, 2007) clearly this is not representative of a system such as Stromboli which has been continuously active for 2000-2500 years (Rosi et al., 2000). This mismatch in mass fluxes could be resolved if the lateral interface position between the ascending and descending magmas and their velocities were to vary with pressure. The experimental studies of exchange flow have shown that the flow regime of an exchange flow depends only on the viscosity ratio between the ascending and descending fluids (Stevenson and Blake, 1998; Huppert and Hallworth, 2007; Beckett et al., 2011), and so it may be reasonable to hypothesise that SBS flow with a transition to CAF would still be observed in this system.

In this study we have assumed a cylindrical conduit, which is clearly an oversimplification. Analysis of seismic signals indicates a transition from a shallow conduit to an inclined dike structure at a depth of 2000-3000 m below the craters (Chouet et al., 2003). We note that the solution to the viscous flow equations in a slot is analogous to those in a pipe (Palma et al., 2011). It seems reasonable therefore to hypothesise, by analogy, that for the viscosity ratios $\gtrsim 100$ that we find for Stromboli magmas deeper than about $3 \mathrm{~km}$, exchange flow in such dyke-like structures would, as in cylindrical pipe-like structures, be characterised by less viscous, vesiculating magma rising up the centre of the slot surrounded by more viscous, degassed and crystallised magma sinking along the dike walls. It would be a useful future experimental study to observe laminar exchange flow in a slot in order to test this hypothesis. A further complication that has yet to be explored experimentally is that several vents are persistently active at Stromboli which may be connected at some depth (e.g. Chouet et al., 2003). It would be interesting to establish whether individual conduits act independently or are affected by their neighbours, and whether the presence of multiple shallow conduits affects the geometry of the deeper exchange flow.

\section{Acknowledgements}

FMB was supported by a studentship funded by the School of Earth Sciences (University of Bristol) and a NERC studentship NE/H525111/ 1, MB, HMM, MP, JCP and FW were supported by NE/F004222/1, and ACR is supported by Royal Society University Fellowship. We thank L. Caricchi and S. Mueller for a stimulating discussion on the rheology of 3-phase fluids, and J. Blundy for a stimulating discussion on melt inclusion trends.

\section{References}

Ahrens, T.J., 1995. Mineral Physics and Crystallography: A Handbook of Physical Constants. American Geophysics Union, Washington DC

Allard, P., 1997. Endogenous magma degassing and storage at Mount Etna. Geophys. Res. Lett. 24, 2219-2222.

Allard, P., Carbonnelle, J., Métrich, N., Loyer, H., Zettwoog, P., 1994. Sulphur output and magma degassing budget of Stromboli volcano. Nature 47, 326-330.

Allard, P., Aiuppa, A., Burton, M., Caltabiano, T., Federico, C., Salerno, G., La Spina, A., 2008. Crater gas emissions and the magma feeding system of Stromboli volcano. In Calvari, S., Inguaggiato, S., Puglisi, G., Ripepe, M., Rosi, M. (Eds.), The Stromboli Volcano: An Integrated Study of the 2002-2003 Eruption. Geophysical Monograph Series, 182.

Asimow, P.D., Ghiorso, M.S., 1998. Algorithmic modifications extending MELTS to calculate subsolidus phase relations. Am. Mineral. 83, 1127-1131.

Beckett, F.M., Mader, H.M.,Phillips, J.C., Rust, A.C., Witham, F., 2011. An experimental study of low Reynolds number exchange flow of two Newtonian fluids in a vertical pipe. J. Fluid Mech. 682, 652-670.

Blundy, J., Cashman, K.V., Rust, A.C., Witham, F., 2010. A case for $\mathrm{CO}_{2}$-rich arc magmas Earth Planet. Sci. Lett. 290, 289-301.

Burton, M., Allard, P., Muré, F., La Spina, A., 2007a. Magmatic gas composition reveals the source depth of slug driven Strombolian explosive activity. Science 317, 227-230.

Burton, M.R., Mader, H.M., Polacci, M., 2007b. The role of gas percolation in quiescent degassing of persistently active basaltic volcanoes. Earth Planet. Sci. Lett. 264 46-60.

Burton, M.R., Caltabiano, T., Murè, F., Salerno, G., Randazzo, D., 2009. $\mathrm{SO}_{2}$ flux from Stromboli during the 2007 eruption: results from the FLAME network and traverse measurements. J. Volcanol. Geotherm. Res. 182, 214-220.

Caricchi, L., Burlini, L.,Ulmer, P.,Gerya, T.,Vassalli, M.,Papale, P., 2007. Non-Newtonian rheology of crystal-bearing magmas and implications for magma ascent dynamics. Earth Planet. Sci. Lett. 264, 402-419.

Chouet, B.,Dawson, P.,Ohminato, T.,Martini, M.,Saccorotti, G.,Giudicepietro, F.,De Luca, G Milana, G., Scarpa, R., 2003. Source mechanisms of explosions at Stromboli Volcano Italy, determined from moment-tensor inversions of very-long-period data. J. Geophys. Res. 108 (B1), 2019.

Costa, A., 2005. Viscosity of high crystal content melts: dependence on solid fraction. Geophys. Res. Lett. 32, L22308.

Costa, A., Caricchi, L., Bagdassarov, N., 2009. A model for the rheology of particle-bearing suspensions and partially molten rocks. Geochem. Geophys. Geosyst. 10, Q03010. 
Di Carlo, I.D.A.,Pichavant, M.,Rotolo, S.G.,Scaillet, B., 2006. Experimental crystallization of a high-K arc basalt: the golden pumice, Stromboli volcano (Italy). J. Petrol. 368 $1317-1343$.

Dixon, E.J., 1997. Degassing of alkalic basalts. Am. Mineral. 82, 368-378.

Dixon, J.E.,Stolper, E.M., 1995. An experimental study of water and carbon dioxide solubilities in mid-ocean ridge basaltic liquids. Part II: applications to degassing. J. Petrol. 36, 1633-1646.

Francalanci, L., Tommasini, S., Conticelli, S.,Davies, G.R., 1999. Sr isotope evidence for short magma residence time for the 20th century activity at Stromboli volcano, Italy. Earth Planet. Sci. Lett. 167, 61-69.

Francalanci, L., Tommasini, S., Conticelli, S., 2004. The volcanic activity of Stromboli in the 1906-1998 AD period: mineralogical, geochemical and isotope data relevant to the understanding of the plumbing system. J. Volcanol. Geotherm. Res. 131, 179-211.

Francalanci, L., Davies, G., Lustenhouwer, W., Tommasini, S., Mason, P.R.D., Conticelli, S., 2005. Intra-grain Sr isotope evidence for crystal recycling and multiple magma reservoirs in the recent activity of Stromboli volcano, southern Italy. J. Petrol. 46, 1997-2021.

Ghiorso, M.S.,Sack, R.O., 1995. Chemical mass transfer in magmatic processes IV. A revised and internally consistent thermodynamic model for the interpolation and extrapolation of liquid-solid equilibria in magmatic systems at elevated temperatures and pressures. Contrib. Mineral. Petrol. 119, 197-212.

Giordano, D., Russell, J.K., Dingwell, D.B., 2008. Viscosity of magmatic liquids: a model Earth Planet. Sci. Lett. 271, 123-134.

Harris, A.J.L., Stevenson, D.S., 1997. Magma budgets and steady-state activity of Vulcano and Stromboli. Geophys. Res. Lett. 24, 1043-1046.

Hui, H.,Zhang, Y., 2007. Toward a general viscosity equation for natural anhydrous and hydrous silicate melts. Geochim. Cosmochim. Acta 71, 403-416.

Huppert, H.E., Hallworth, M.A., 2007. Bi-directional flows in constrained systems. J. Fluid Mech. 578, 95-112.

Kazahaya, K., Shinohara, H., Saito, G., 1994. Excessive degassing of Izu-Oshima volcano: magma convection in a conduit. Bull. Volcanol. 56, 207-216.

Kerswell, R.R., 2011. Exchange flow of two immiscible fluids and the principle of maximum flux. J. Fluid Mech. 682, 132-159.

Landi, P., Métrich, N., Bertagnini, A., Rosi, M., 2004. Dynamics of magma mixing and degassing recorded in plagioclase at Stromboli (Aeolian Archipelago, Italy). Contrib. Mineral. Petrol. 147, 213-227.

Landi, P.,Métrich, N.,Bertagnini, A.,Rosi, M., 2008. Recycling and 're-hydration' of degassed magma inducing transient dissolution/crystallization events at Stromboli (Italy). J. Volcanol. Geotherm. Res. 174, 325-336.

Lautze, N.,Houghton, B., 2007. Linking variable explosion style and magma textures during 2002 at Stromboli volcano, Italy. Bull. Volcanol. 69, 445-460.

Lesne, P., Kohn, S., Blundy, J.D., Witham, F., Botcharnikov, R., Behrens, H., 2011. Experimental simulation of closed-system degassing in the system basalt- $\mathrm{H}_{2} \mathrm{O}-\mathrm{CO}_{2}-\mathrm{S}-\mathrm{Cl}$. J. Petrol. 52, 1737-1762.

Llewellin, E.W.,Manga, M., 2005. Bubble suspension rheology and implications for conduit flow. J. Volcanol. Geotherm. Res. 143, 205-217.

Mader, H.M.,Llewellin, E.W.,Mueller, S.P., 2013. The rheology of two-phase magmas: a review and analysis. J. Volcanol. Geotherm. Res. 257, 135-158.

Métrich, N.,Bertagnini, A.,Landi, P., Rosi, M., 2001. Crystallization driven by decompression and water loss at Stromboli volcano (Aeolian Islands, Italy). J. Petrol. 42, 1471-1490.
Métrich, N.,Bertagnini, A., Di Muro, A., 2010. Conditions of magma storage, degassing and ascent at Stromboli: new insights into the volcano plumbing system with inferences on the eruptive dynamics. J. Petrol. 51, 603-626.

Molina, I.,Burgisser, A., Oppenheimer, C., 2012. Numerical simulations of convection in crystal-bearing magmas: a case study of the magmatic system at Erebus, Antarctica. J. Geophys. Res. 117, B07209.

Mueller, S., Llewellin, E.W., Mader, H.M., 2010. The rheology of suspensions of solid particles. Proc. R. Soc. Lond. 466, 1201-1228.

Murase, T., McBirney, A.R., 1973. Properties of some common igneous rocks and their melts at high temperatures. Geol. Soc. Am. Bull. 84, 3563-3592.

Newman, S., Lowenstern, J.B., 2002. VolatileCalc: a silicate melt- $\mathrm{H}_{2} \mathrm{O}-\mathrm{CO}_{2}$ solution model written in Visual Basic for excel. Comput. Geosci. 28, 597-604.

Pal, R., 2002. Rheological behaviour of bubble-bearing magmas. Earth Planet. Sci. Lett. 207, $165-179$.

Pal, R., 2004. Rheological constitutive equation for bubbly suspensions. Ind. Eng. Chem. Res. 43, 5372-5379.

Palma, J.L.,Blake, S.,Calder, E.S., 2011. Constraints on the rates of degassing and convection in basaltic open vent volcanoes. Geochem. Geophys. Geosyst. 12, 11

Papale, P., Moretti, R., Barbato, D., 2006. The compositional dependence of the saturation surface of $\mathrm{H}_{2} \mathrm{O}+\mathrm{CO}_{2}$ fluids in silicate melts. Chem. Geol. 229, 78-95.

Persikov, E., Bukhtiyarov, P., Nekrasov, A., 2010. Water diffusion in basalt and andesite melts under high pressures. Geochem. Int. 48, 213-225.

Pichavant, M., Di Carlo, I., Le Gac, Y., Rotolo, S., Scaillet, B., 2009. Experimental constraints on the deep magma feeding system at Stromboli volcano, Italy. J. Petrol. 50, 601-624.

Pitzer, K.S.,Sterner, S.M., 1994. Equations of state valid continuously from zero to extreme pressures for $\mathrm{H}_{2} \mathrm{O}$ and $\mathrm{CO}_{2}$. J. Chem. Phys. 101, 3111-3116.

Polacci, M., Baker, D.R.,Mancini, L., Favretto, S.,Hill, R.J., 2009. Vesiculation in magmas from Stromboli and implications for normal Strombolian activity and paroxysmal explosions in basaltic systems. J. Geophys. Res. 114, B01206.

Richet, P.,Whittington, A.,Holtz, F.,Behrens, H.,Ohlhorst, S.,Wilke, M., 2000. Water and the density of silicate glasses. Contrib Mineral Petrol 138, 337-347.

Ripepe, M.,Harris, A.J.L.,Carneil, R., 2002. Thermal, seismic and infrasonic evidences of variable degassing rates at Stromboli volcano. J. Volcanol. Geotherm. Res. 118, 285-297.

Ripepe, M.,Marchetti, E.,Ulivieri, G., 2007. Infrasonic monitoring at Stromboli volcano during the 2003 effusive eruption: insights on the explosive and degassing process of an open conduit system. J. Geophys. Res. 112, B09207.

Rosi, M.,Bertagnini, A.,Landi, P., 2000. Onset of the persistent activity at Stromboli Volcano (Italy). Bull. Volcanol. 62, 294-300.

Rust, A.C., Manga, M., 2002. Effects of bubble deformation on the viscosity of dilute suspensions. J. Non-Newtonian Fluid Mech. 104, 53-63.

Stevenson, D.S., Blake, S., 1998. Modelling the dynamics and thermodynamics of volcanic degassing. Bull. Volcanol. 60, 307-317.

Witham, F., 2011a. Conduit convection, magma mixing, and melt inclusion trends at persistently degassing volcanoes. Earth Planet. Sci. Lett. 301, 345-352.

Witham, F., 2011b. Conduit convection, magma mixing, and melt inclusion trends at persistently degassing volcanoes. Reply to comment by Métrich et al. (2011). Earth Planet Sci. Lett. 306, 309-311.

Witham, F.,Blundy, J., Kohn, S.C., Lesne, P.,Dixon, J.,Churakov, C., Botcharnikov, 2012. SolEx: a model of mixed COHSCL-volatile solubilities and exsolved gas compositions in basalt. Comput. Geosci. 45, 87-97. 